\title{
Histone methyltransferase MLL4 controls myofiber identity and muscle performance through MEF2 interaction
}

\author{
Lin Liu, ${ }^{1,2}$ Chenyun Ding, ${ }^{1,2}$ Tingting Fu, ${ }^{1,2}$ Zhenhua Feng, ${ }^{3}$ Ji-Eun Lee, ${ }^{4}$ Liwei Xiao, ${ }^{1,2}$ Zhisheng Xu, ${ }^{1,2}$ Yujing Yin, ${ }^{1,2}$ Qiqi Guo, ${ }^{1,2}$ \\ Zongchao Sun, ${ }^{1,2}$ Wanping Sun, ${ }^{1,2}$ Yan Mao, ${ }^{1,2}$ Likun Yang, ${ }^{1,2}$ Zheng Zhou, ${ }^{1,2}$ Danxia Zhou, ${ }^{1,2}$ Leilei Xu, ${ }^{3}$ Zezhang Zhu, ${ }^{3}$ \\ Yong Qiu, ${ }^{3}$ Kai Ge, ${ }^{4}$ and Zhenji Gan ${ }^{1,2}$ \\ 1State Key Laboratory of Pharmaceutical Biotechnology and MOE Key Laboratory of Model Animals for Disease Study, Department of Spine Surgery, Nanjing Drum Tower Hospital, The Affiliated Hospital \\ of Nanjing University Medical School, Model Animal Research Center, Nanjing, China. ${ }^{2}$ Chemistry and Biomedicine Innovation Center (ChemBIC), Nanjing University, Nanjing, China. ${ }^{3}$ Department of Spine \\ Surgery, Nanjing Drum Tower Hospital, Affiliated Hospital of Nanjing University Medical School, Nanjing, China. ${ }^{4}$ Adipocyte Biology and Gene Regulation Section, National Institute of Diabetes and Digestive \\ and Kidney Diseases, NIH, Bethesda, Maryland, USA.
}

\begin{abstract}
Skeletal muscle depends on the precise orchestration of contractile and metabolic gene expression programs to direct fibertype specification and to ensure muscle performance. Exactly how such fiber type-specific patterns of gene expression are established and maintained remains unclear, however. Here, we demonstrate that histone monomethyl transferase MLL4 (KMT2D), an enhancer regulator enriched in slow myofibers, plays a critical role in controlling muscle fiber identity as well as muscle performance. Skeletal muscle-specific ablation of MLL4 in mice resulted in downregulation of the slow oxidative myofiber gene program, decreased numbers of type I myofibers, and diminished mitochondrial respiration, which caused reductions in muscle fatty acid utilization and endurance capacity during exercise. Genome-wide ChIP-Seq and mRNA-Seq analyses revealed that MLL4 directly binds to enhancers and functions as a coactivator of the myocyte enhancer factor 2 (MEF2) to activate transcription of slow oxidative myofiber genes. Importantly, we also found that the MLL4 regulatory circuit is associated with muscle fiber-type remodeling in humans. Thus, our results uncover a pivotal role for MLL4 in specifying structural and metabolic identities of myofibers that govern muscle performance. These findings provide therapeutic opportunities for enhancing muscle fitness to combat a variety of metabolic and muscular diseases.
\end{abstract}

\section{Introduction}

Muscle fitness is a key determinant of human health and disease. Muscle performance and fatigability are determined by the structural and metabolic properties of the specialized myofibers (1-3). Myofibers differ markedly in their contractile and energy metabolism functions and can generally be classified as slow-twitch (type I) and fast-twitch (type II). Type I myofibers are rich in mitochondria, rely largely on mitochondrial oxidative metabolism, and are resistant to fatigue $(4,5)$, whereas type II myofibers generally contain fewer mitochondria, have lower oxidative capacity, are fatigue sensitive, and can be subclassified as type IIa, IIx, or IIb in rodents based on the type of myosin heavy chain (MHC) isoform expressed $(4,5)$. Myofibers maintain remarkable plasticity for undergoing metabolic and structural remodeling during development and in response to physiological stimuli and systemic diseases. Exercise enhances muscle performance and endurance by increasing the proportion of slow oxidative fibers and by promot-

Authorship note: $L L$ and $C D$ are co-first authors and contributed equally to this work. Conflict of interest: The authors have declared that no conflict of interest exists. Copyright: ( 2020 , American Society for Clinical Investigation.

Submitted: January 8, 2020; Accepted: May 29, 2020; Published: August 4, 2020. Reference information: J Clin Invest. 2020;130(9):4710-4725

https://doi.org/10.1172/JCl136155. ing the capacity of mitochondria to burn fatty acids and glucose as their chief fuels (6-10). Conversely, reduced muscle fitness, including a shift away from slow oxidative fibers and decreased mitochondrial oxidative capacity, is a common consequence of a variety of human illnesses, including metabolic disorders and muscular diseases $(3,7,11,12)$.

Myofibers depend on the precise orchestration of contractile and metabolic gene expression programs to direct fiber-type specification and ensure muscle performance. Exactly how such fiber-type-specific patterns of gene expression are established and maintained remains unclear, however. Previous studies have identified multiple transcriptional factors, such as nuclear receptor PPARs, ERRs, and myocyte enhancer factor 2 (MEF2), along with coregulators PGC-1s, NCoR1, and HDACs in the regulation of diverse metabolic and structural gene expression in muscle (13-21). As a critical step for gene activation and the primary site of the gene-environment interaction, enhancer activation is increasingly recognized as a key layer of regulation for adapting gene transcription to environmental cues. Active enhancers are marked by the presence of monomethylated histone $\mathrm{H} 3$ lysine 4 (H3K4me1) and acetylated histone H3 lysine 27 (H3K27ac) (22, 23). Whether and how the epigenetic regulation of enhancer activation orchestrates muscle fiber-type-specific patterns of gene expression are unclear. 
Mixed-lineage leukemia 4 (MLL4/KMT2D), a major histone $\mathrm{H} 3$ lysine 4 (H3K4) monomethyl transferase, is an essential histone writer for enhancer activation (24-28). MLL4 has been shown to colocalize with cell type-specific transcription factors to establish active enhancers during cell differentiation in multiple cell types (26-29). Loss of MLL4 prevents the establishment of $\mathrm{H} 3 \mathrm{~K} 4 \mathrm{me} 1$ and H3K27ac signatures on enhancer regions, leading to significant defects in enhancer activation and, consequently, gene transcription $(26-28,30)$. Studies in mouse models further highlighted the importance of MLL4 in regulating a wide range of biological processes, including embryonic development, metabolic homeostasis, and cancer (27, 29, 31-33). Moreover, frequent mutations in MLL4 have been implicated in several human genetic diseases, including Kabuki syndrome, congenital heart disease, and various types of cancer (33-35).

As a critical regulator of cell differentiation and cell-fate transition, MLL4 has been documented as directing embryonic muscle and fat tissue development $(27,29)$. However, it has yet to be explored whether MLL4 has a role in adult skeletal muscle. In this study, we generated 3 independent skeletal muscle-specific Mll4KO mouse lines and found that slow myofiber-enriched MLL4 is required for slow oxidative type I fiber formation to ensure muscle endurance. Mice with muscle-specific ablation of MLL4 exhibited marked downregulation of the slow oxidative myofiber gene program and had a decreased number of type I myofibers and amount of mitochondrial activity, resulting in reduced muscle fatty acid utilization and endurance capacity during exercise. Mechanistically, we demonstrated that MLL4 drives the slow oxidative type I fiber program in cooperation with MEF2. Our studies also strongly suggest that the MLL4-dependent regulation of the type I fiber program is operational in human muscle.

\section{Results}

Slow myofiber-enriched MLL4 is required for type I fiber formation. As an initial step for exploring the potential function of MLL4 in adult muscle, we examined the expression patterns of MLL4 in different muscle types from adult WT mice. Comparison of slow fiber-dominant soleus muscle to fast fiber-enriched white vastus lateralis (WV) by Western blot revealed that MLL4 protein is expressed preferentially ( 2 -fold) in soleus muscle (Figure $1 \mathrm{~A})$. In contrast, the protein levels of EZH2 and SUZ12, key components of the polycomb repressive complex 2 , were markedly decreased in soleus muscle compared with those in WV (Figure 1A). These results suggest that MLL4 may activate the expression of slow fiber genes in slow myofibers.

To directly examine the role of MLL4 in skeletal muscle, we generated 2 independent skeletal muscle-specific $M l l 4$-KO mouse lines using $M l l 4^{f l / f l}$ and $M l l 4 S E T^{f / f l}$ mice. In the $M l l 4^{f / f l}$ strain, the exons 16-19 were flanked by 2 loxP sites (27), while in the $M l l 4 S E T^{f / f l}$ strain, the exons 50 and 51 encoding the enzymatic SET domain of MLL4 were floxed (29). Mll4 $4^{f / f l}$ and Mll4SET $T^{f / f l}$ mice were bred with mice expressing Cre in postnatal skeletal muscle, in which Cre expression is under the control of the muscle creatine kinase $(M c k)$ promoter (36), to achieve deletion of Mll4 in both fast- and slow-twitch muscle fiber types (referred to as Mll4-mKO and Mll4SET-mKO, respectively). This resulted in efficient postnatal deletion of $M l l 4$ in skeletal muscle. Consistent with previous- ly published data $(37,38)$, efficient ablation of Mll4 mediated by Mck-Cre did not occur until approximately 7 days after birth (Supplemental Figure 1A; supplemental material available online with this article; https://doi.org/10.1172/JCI136155DS1). As expected, the expression of Mll4 mRNA and protein levels were markedly reduced in fast and slow muscles from both muscle-specific Mll4KO mouse lines (Figure 1B and Supplemental Figure 1, B-E). Notably, deleting the enzymatic SET domain destabilized endogenous MLL4 protein in muscle (Figure 1B and Supplemental Figure 1D), which is consistent with recently published data (29). Both Mll4$\mathrm{mKO}$ and Mll4SET-mKO mice were born at normal Mendelian ratios and did not exhibit an overt metabolic phenotype compared with WT littermates on standard chow. These phenotypes included similar body weight, food intake, locomotor activity, energy expenditure, and muscle weight (Supplemental Figure 2 and Supplemental Figure 3). This indicates that postnatal ablation of Mll4 neither affects muscle differentiation nor development. Examination of myofiber cross-sectional area by histochemical staining revealed that fiber-size distribution was altered in gastrocnemius (GC) muscle from both muscle-specific Mll4-KO mouse lines, with a shift toward an increased cross-sectional area relative to that of WT controls (Figure 1C and Supplemental Figure 3M). Muscle phenotyping and transcriptional profiling revealed that fibertype programs were substantially regulated in the muscle-specific Mll4-KO mouse lines. Expression of the gene encoding the major slow-twitch type I myosin isoform MHC1 (Myh7 gene) and slowtwitch troponin genes (Tnni1, Tnnc1, and Tnnt1) was reduced in both Mll4-mKO and Mll4SET-mKO GC muscle (Figure 1D). In contrast, expression of the fast-twitch troponin gene (Tnnt3) was increased in muscle from the $2 \mathrm{Mll4}$-KO mouse lines (Figure 1D). Similar observations were made in soleus and WV muscles from the Mll4SET-mKO mice (Supplemental Figure 4). Consistent with the gene expression results, MHC1 immunofluorescence staining revealed a marked reduction in the number of type I fibers in several muscle groups from both Mll4-mKO and Mll4SET-mKO mice (Figure 1, E and F). Notably, the Mll4SET-mKO mouse line did not exhibit overt muscle phenotypes in young mice at P10 (Supplemental Figure 5), suggesting that the observed results were not a consequence of changes in muscle development or early fibertype patterning in Mll4SET-mKO mice.

An in vitro primary skeletal myocyte culture system further demonstrated that the MLL4 protein levels were induced during differentiation of myoblasts into mature myotubes (Supplemental Figure 6A). Consistent with the established role of MLL4 in maintaining H3K4me1 levels on active enhancers $(27,28,30)$, the induced expression of slow myosin paralleled the elevated levels of H3K4me1 on the $M y h 7$ enhancer during muscle cell differentiation (Supplemental Figure 6, A and B). An MLL4 loss-of-function study was also conducted in primary skeletal myocyte. We used adenoviral vectors to express Cre or control viruses in Mll4SET $T^{f / f l}$ myoblasts. Cells were then induced to differentiate into myotubes. Adenoviral Cre-mediated $\mathrm{KO}$ of Mll4 in myoblasts resulted in diminished expression of $M y h 7$ and decreased proportion of slow myosin-positive myotubes (Figure 1, G and H). In contrast, fast myosin gene $M y h 4$ expression was not decreased by $M l l 4$ deletion (Figure 1, G and H). Consistent with reduction of $M y h 7$ mRNA in Mll4-KO myotubes, MLL4 deficiency reduced the amounts of 


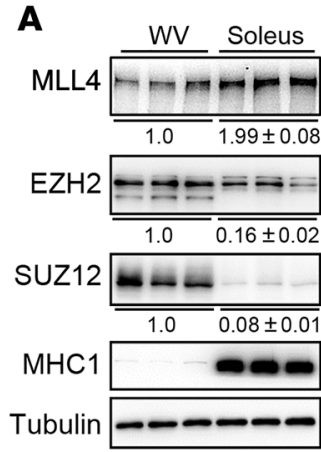

B

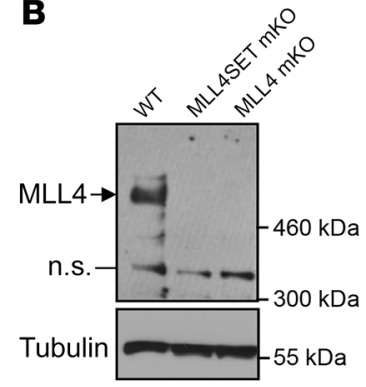

D

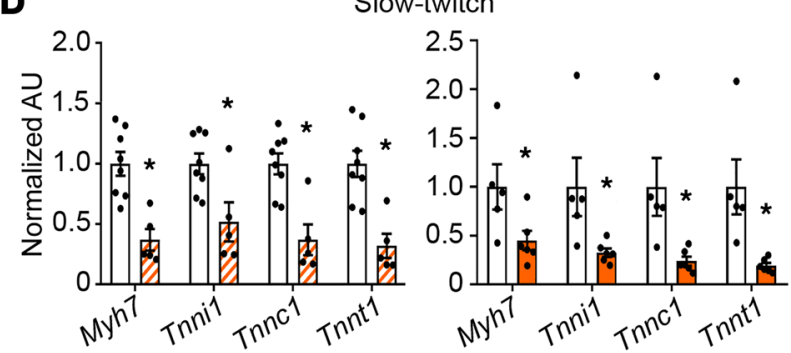

$\mathbf{E}$
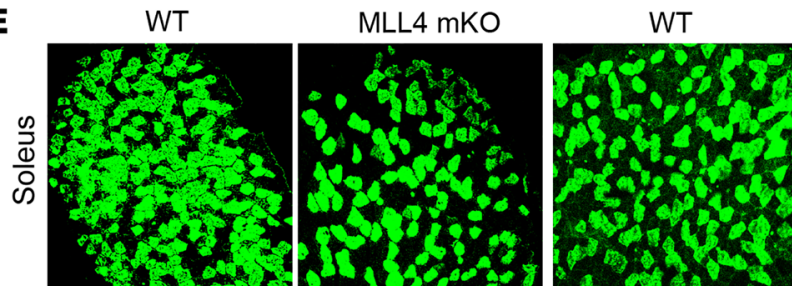

O
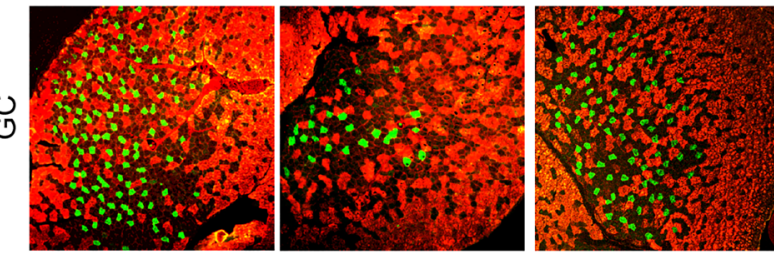

C

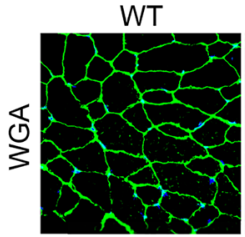

MLL4 mKO
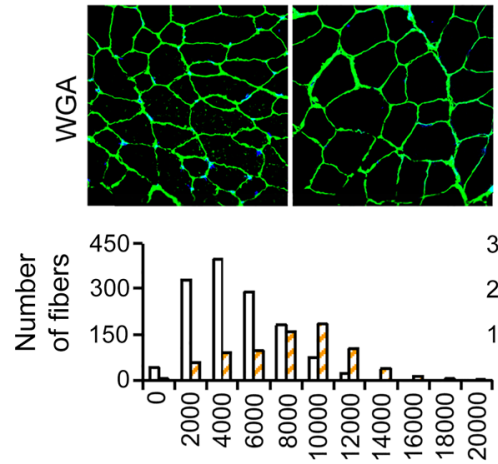

Myofiber cross-sectional area (arbitrary units)

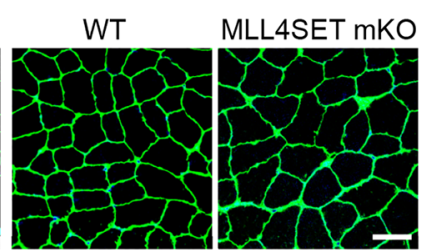
300
$200-1$ 口MLL4SET mKO 100

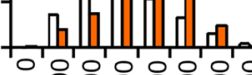

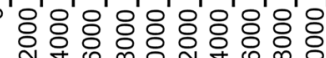
Myofiber cross-sectional area (arbitrary units)

$$
\square \mathrm{MHC} 1 \mathrm{MHC} 2 \mathrm{~b}
$$

G

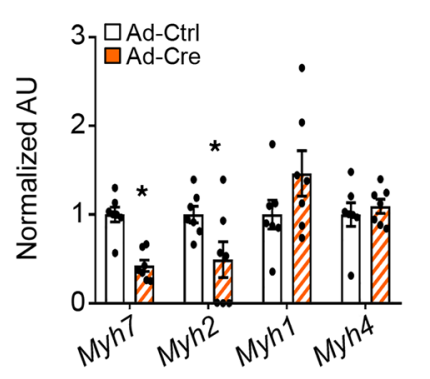

H

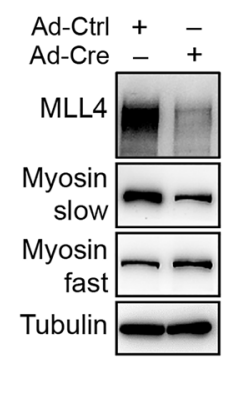

Fast-twitch
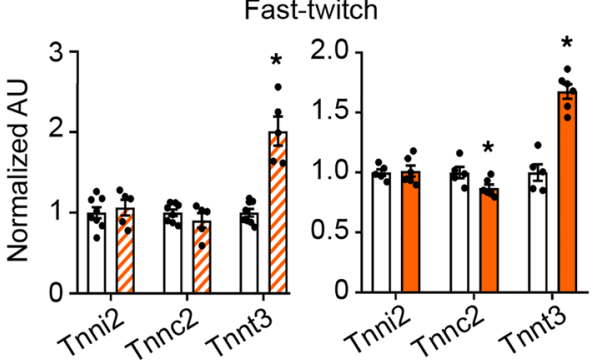

口WT

口MLL4 mKO

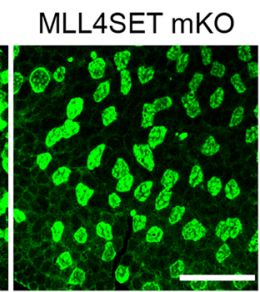

$\begin{array}{ll}\text { F } & \text { gWT } \\ \text { घMLL4 mKO } \\ \text { वMLL4SET mKO }\end{array}$
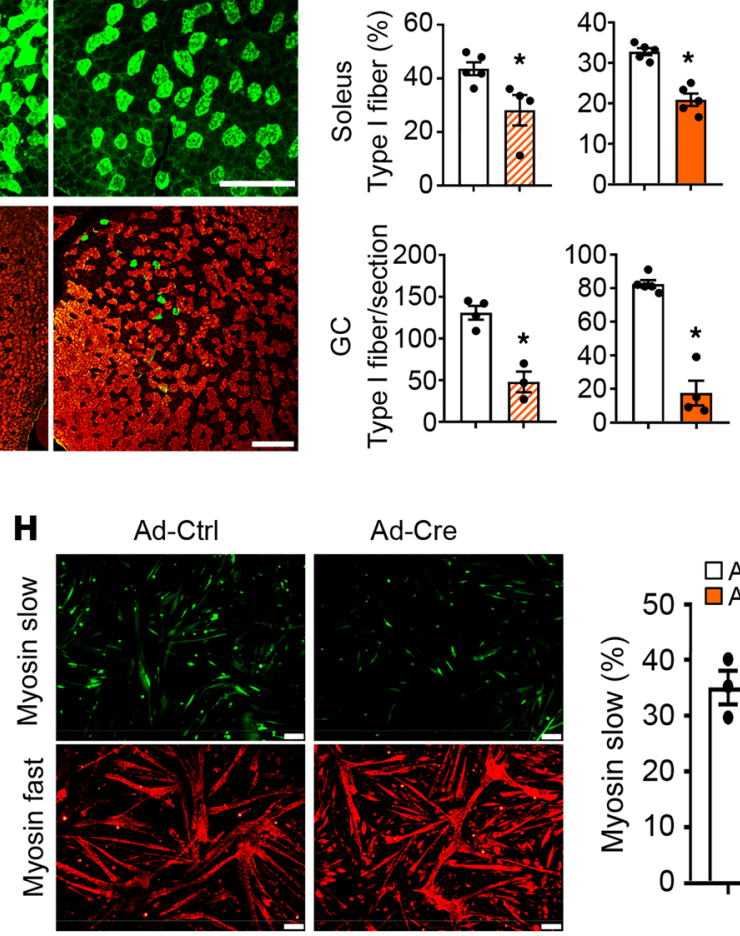

Ad-Cre
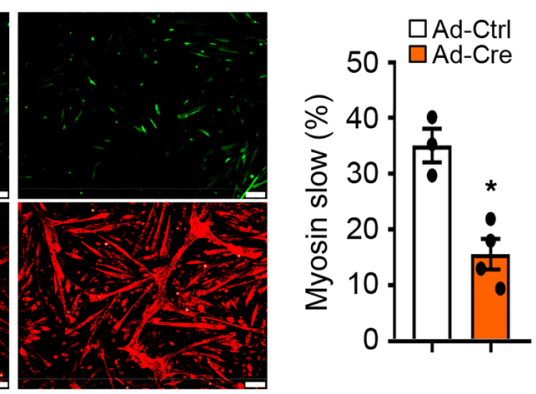
Figure 1. Slow myofiber-enriched MLL4 is required for type I muscle fiber formation. (A) Representative Western blot analysis of protein extracts prepared from WV and soleus muscles of WT mice using indicated antibodies. Quantification of the MLL4/tubulin, EZH2/tubulin, and SUZ12/ tubulin signal ratios normalized $(=1.0)$ to the $W V$ and presented below the corresponding bands. $n=5-6$ mice per group. (B) Representative Western blot analysis of MLL4 expression in GC muscles of indicated mice. n.s., nonspecific band. $n=3$ mice per group. (C) Top: representative WCA staining of GC muscle from 8-week-old male MII4-mKO and MII4SET-mKO mice. Scale bar: $50 \mu \mathrm{m}$. Bottom: cross-sectional areas of GC myofibers were measured by ImageJ. $n=4-5$ mice per group. (D) Expression of slowtwitch myosin gene (Myh7) and representative slow/fast-twitch troponin genes (qRT-PCR) in GC muscle from indicated genotypes. $n=5-8$ mice per group. (E) Cross section of (top) soleus and (bottom) GC muscle from 8-week-old male MII4-mKO and MII4SET-mKO mice stained for MHC1 (green) and MHC2b (red). Scale bars: $250 \mu \mathrm{m}$. (F) Quantification of IF data shown in $\mathbf{E}$. $n=3-5$ mice per group. (G and $\mathbf{H}$ ) Primary myoblasts isolated from MII4SET $T^{f / f l}$ mice were infected with an adenovirus overexpressing Cre or control virus (Ctrl), followed by differentiation into myotubes. (C) Results of qRT-PCR and Western blot analysis in skeletal myotubes. $n=3$ independent experiments. (H) Left: IF staining of skeletal myotubes was performed using antibodies directed against myosin-slow or myosin-fast. Scale bars: $100 \mu \mathrm{m}$. Right: quantification of the myosin-slow IF data expressed as mean percentage of total myotubes. $n=3$ independent experiments. Values are represented as mean $\pm \mathrm{SEM}$. ${ }^{*} P<0.05$ vs. corresponding controls, 2-tailed unpaired Student's $t$ test.

H3K4me1 on the $M y h 7$ enhancer (Supplemental Figure 6C). Thus, in vitro manipulation of Mll4 in myocytes provided further evidence that MLL4 exerts control upon slow fiber gene expression. Taken together, these data demonstrate an essential role of MLL4 in the regulation of type I muscle fiber type.

Loss of muscle MLL4 causes reduced running endurance. Muscle fiber-type composition and the capacity to burn fatty acids and glucose are important determinants of muscle performance and endurance, and fast-twitch muscle fibers exhibit a glycolytic burst metabolism and are more susceptible to endurance exerciseinduced fatigue (5). We next sought to determine the physiological impact of muscle MLL4 deficiency. To rule out possible effects mediated by MLL4 in the heart on exercise phenotypes, Mll4SET $T^{l / f l}$ mice were bred with human skeletal actin Cre (HSA-Cre) mice to establish another skeletal muscle-specific deletion of the Mll4 alleles. As expected, the protein expression of Mll4 was dramatically decreased in multiple muscle types, but not in the heart, in $M l l 4 S E T^{f / f l} H S A$-Cre mice (Supplemental Figure 7A). In addition, MHC1-positive fibers were markedly reduced in Mll4SET $T^{f / f l}$ HSA-Cre GC muscle (Figure 2, A and B, and Supplemental Figure $7 \mathrm{~B})$. We next assessed acute running endurance performance in the Mll4SET $T^{f / f l} H S A$-Cre mouse line using a run-to-exhaustion protocol on a motorized treadmill. Consistent with the observed alterations in muscle fiber-type proportion, Mll4SET ${ }^{f / f l} H S A$-Cre mice could run for significantly shorter times and distances ( 30\%) compared with WT littermates (Figure 2C).

To further evaluate muscle fuel utilization during exercise, WT or Mll4SET $T^{f / f l} H S A$-Cre mice were first subjected to a forced maximal exercise capacity test $\left(\mathrm{VO}_{2 \max }\right.$ test) consisting of increasing speed every 2 minutes at $10^{\circ}$ inclination until exhaustion (Supplemental Figure 8A). Consistent with a shift to muscle glucose utilization, the respiratory exchange ratio (RER) increased with exercise in both Mll4SET $T^{f / f l} H S A$-Cre mice and the WT control group, indicative of a switch to carbohydrates as the chief fuel (Supplemental Figure 8, A-D). Interestingly, in this high-intensity exercise challenge, both genotypes demonstrated similar energy substrate utilization (RER) during the course of exercise, and Mll4SET $T^{f / f l} H S A-C r e$ mice ran distances and achieved maximal speed similarly to their control littermates (Supplemental Figure $8, \mathrm{C}-\mathrm{F}$ ). Mice that performed the $\mathrm{VO}_{2 \max }$ test were then subjected to a low-intensity (endurance type) protocol, during which animals ran at a constant speed of $60 \%$ of their maximal running speed (Supplemental Figure 8G). While RER values increased during the beginning of the exercise test, both WT and Mll4SET $T^{f / f l}$ HSA-Cre mice displayed a drop in RER after approximately 14 minutes of endurance exercise (Supplemental Figure 8, G-I). This decrease indicates a shift in substrate usage from glucose to fat metabolism. Despite no difference in RER during the exercise period, the Mll4SET ${ }^{f / f l} H S A$-Cre mice ran shorter distances compared with the WT control group (Supplemental Figure 8, H-K). These results suggest that muscle MLL4 deficiency resulted in reduced capacity for persistent use of fat as a fuel during endurance exercise. Furthermore, we also measured glucose and fatty acid levels in blood from WT and Mll4SET $T^{f / f l} H S A$-Cre mice before and after endurance exercise. Mll4SET $T^{f / f l} H S A$-Cre mice showed significantly lower blood glucose levels compared with WT controls at baseline and after 80 minutes of endurance exercise (Figure 2D). Conversely, blood triglyceride (TG) and fatty acid levels were significantly higher after exercise in $M l l 4 S E T^{f / f l} H S A-C r e$ mice (Figure 2E). Notably, blood ketone body $\beta$-hydroxybutyrate levels mirrored the changes of blood fatty acids, as they were also increased in Mll4SET $T^{f / f l} H S A$-Cre mice at basal conditions or after exercise (Figure 2E). Together, these results demonstrate a change in slow type I fibers and muscle metabolism in the absence of muscle MLL4 that compromises running endurance.

MLL4 coordinates gene programs controlling muscle contraction and energy metabolism. Type I myofibers depend on the precise orchestration of slow contractile and oxidative metabolic gene expression to ensure muscle endurance. To more thoroughly analyze the fiber-type-specific patterns of gene expression changes that result from loss of muscle MLL4, we performed RNA-Seq analysis on mRNA isolated from GC muscles of Mll4SET-mKO mice and littermate controls. We found that MLL4 regulated a total of 1000 genes in skeletal muscles, with 447 up- and 553 downregulated, respectively (Figure $3 \mathrm{~A}$ ). In addition to the key slow-twitch MHC gene $M y h 7$ and troponin complex described above (Figure 1D), gene ontology (GO) analysis of MLL4-regulated genes also revealed significant enrichment in fiber-typespecific isoforms of sarcomeric components as well as ion channels involved in excitation-contraction coupling (Figure 3B). The comparative RNA-profiling strategy revealed extensive fiber-type switching and novel myofiber-specific gene expression. As shown in Figure 3C, a broad array of contraction-related genes that differ between muscle fiber type were altered in Mll4SET-mKO muscle (Figure 3C). Real-time PCR confirmed that the expression of many genes encoding slow contractile proteins ( $M y l 2, M y l 3$, Tpm3, and Myom3) was reduced in the GC muscles of Mll4SET-mKO mice compared with those of WT controls (Figure 3D). The expression of slow fiber calcium-handling genes (Atp2a2, Casq2, Smtnl1, and 
A

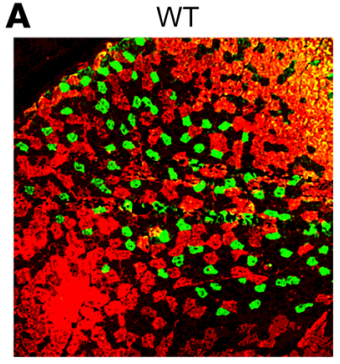

C

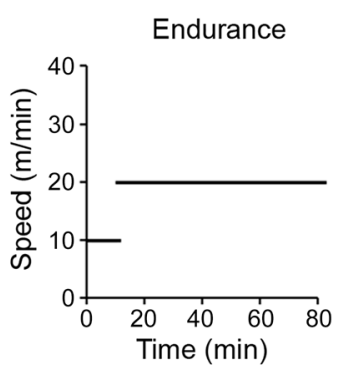

MIILSET ${ }^{\text {f/fi/HSA-Cre }}$
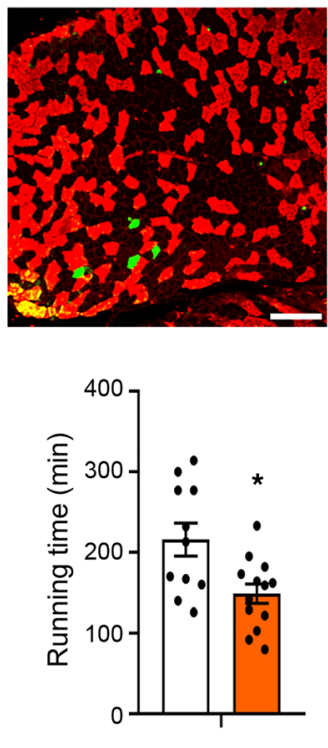

\section{$\square \mathrm{MHC1}$}

$\square \mathrm{MHC} 2 \mathrm{~b}$

B
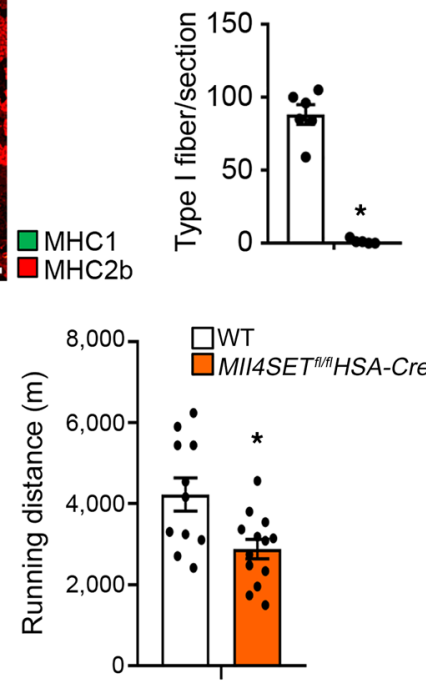

MII4SET IIfH HSA-Cre

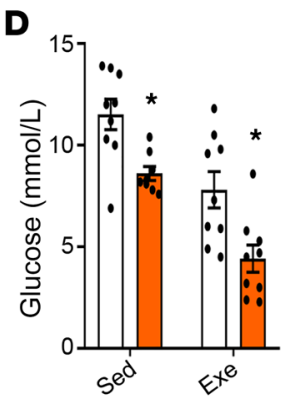

E

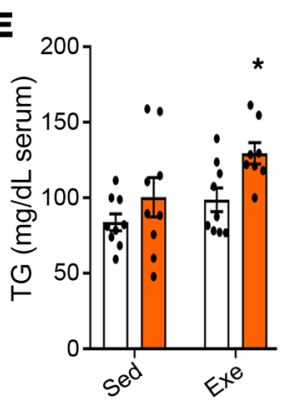

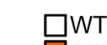

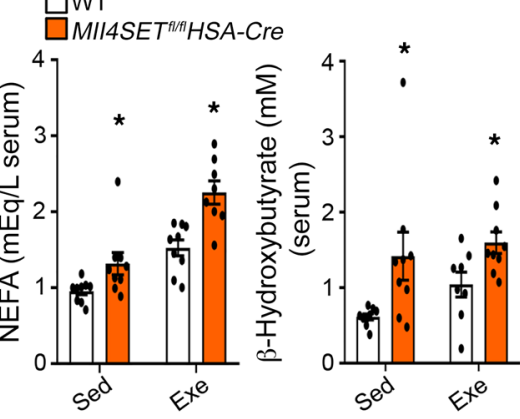

Figure 2. Loss of muscle MLL4 causes reduced running endurance. (A) MHC fiber typing by IF of GC muscle of indicated genotypes. MHC1 (green); MHC2b (red). Representative images are shown. Scale bar: $250 \mu \mathrm{m}$. (B) Quantification of IF data shown in $\mathbf{A}$ expressed as type I fibers per section. $n=5-6$ mice per group. (C) Left: schematic depicts increments of speed over time. Right: bars represent mean running time and distance for 10 -week-old male MII4SET ${ }^{\text {fl/fl}} H S A$-Cre mice and WT on a motorized treadmill. $n=11-13$ mice per group. (D and E) Blood glucose, TC, NEFA, and $\beta$-hydroxybutyrate levels in mice of indicated genotypes at rest or after 80 minutes of exercise. $n=8-9$ mice per group. Values are represented as mean \pm SEM. ${ }^{*} P<0.05$ vs. corresponding WT controls, 2-tailed unpaired Student's $t$ test.
Myoz2) was also reduced in the Mll4SET-mKO muscles compared with those in WT controls (Figure 3D). Moreover, we also found a decreased expression of genes associated with fatty acid and glucose metabolism (Cpt1b, Slc27a1, Fabp3, Dgat2, Fads6, Phyhd1, $L d h b$, and Ppp1r1a) in Mll4SET-mKO muscle (Figure 3D). The expected lactate dehydrogenase (LDH) isoenzyme activity shifts due to changes in $L d h b$ expression were confirmed by gel-activity studies (Figure 3E). To further assess the metabolic effects of muscle MLL4, mitochondrial respiration rates were determined in the extensor digital longus (EDL) muscle of Mll4SET-mKO mice and WT controls using pyruvate or palmitoylcarnitine as substrates. State 3 (maximal ADP stimulated) respiration rates were significantly lower in Mll4SET-mKO muscle compared with that of WT controls (Figure 3F). Moreover, measurement of oxygen consumption rate (OCR) using an extracellular flux analyzer also revealed that Mll4SET-KO myotubes had a reduced OCR in the presence of uncoupler FCCP, a sign of reduced maximal mitochondrial respiratory capacity (Figure 3G), whereas no difference in the extracellular acidification rate (ECAR) was observed in Mll4SET-KO myotubes compared with Mll4SET $T^{f / f l}$ controls (data not shown). Together, these results demonstrate that MLL4 programs type I muscle metabolism by coordinate regulation of gene programs controlling muscle contraction and energy metabolism.

$M L L 4$ drives the slow oxidative muscle fiber program in cooperation with MEF2. To define the mechanism involved in the acti- vation of slow oxidative muscle fiber gene programs by MLL4, we examined the possibility that MLL4 regulates factors known to regulate slow myofiber gene expression. Esrrg mRNA levels were modestly increased and Ppard mRNA levels were lower in Mll4SET-mKO muscles (Supplemental Figure 9), whereas Fnip1, Ppargc1a, Ppargc1b, Ppara, Esrra, and Esrrb gene expression were not changed in Mll4SET-mKO muscles (Supplemental Figure 9). These results led us to the hypothesis that MLL4 may act as a direct activator of slow-myofiber gene expression.

We next sought to examine the genome-wide MLL4 occupancy on WT mouse muscle chromatin by ChIP coupled with high-throughput DNA sequencing (ChIP-Seq). As a control for specificity, ChIP-Seq experiments were also performed in Mll4-mKO muscle; 9403 high-confidence MLL4 genomic binding regions were obtained by filtering out nonspecific signals observed in MLL4-deficient muscle (Supplemental Figure 10, $\mathrm{A}-\mathrm{C}$ ). MLL4-binding sites in skeletal muscle were predominantly located in the intergenic, intronic, and promoter regions (Supplemental Figure 10D), which is consistent with our previous MLL4 ChIP-Seq results in myocytes (27). Correlating the MLL4 cistrome in muscle and myocytes with the global mRNA changes upon muscle Mll4 deletion revealed that approximately $49 \%$ of MLL4-regulated genes were directly bound by MLL4 (based on the genes nearest to the MLL4-binding peaks) (Figure 4A). Furthermore, GO analysis revealed that the 492 directly regulated genes were 
A
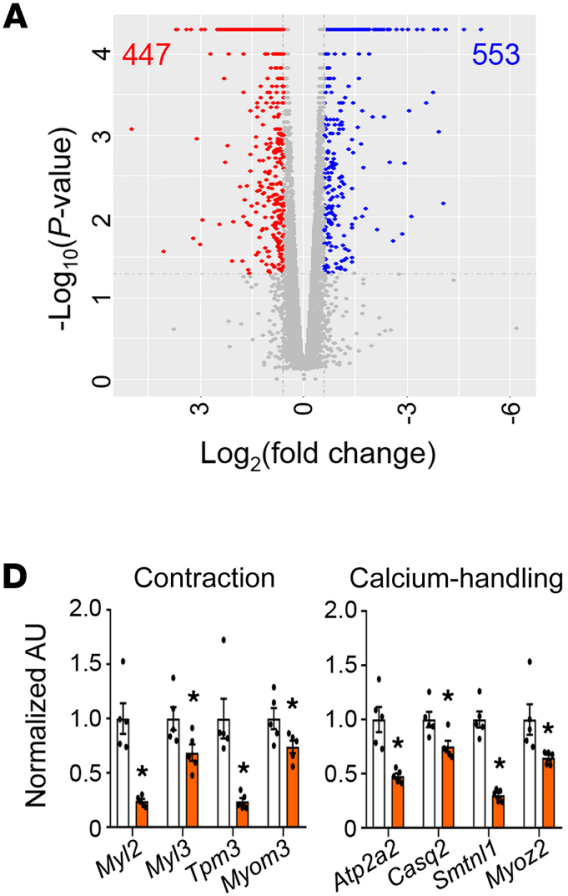

E

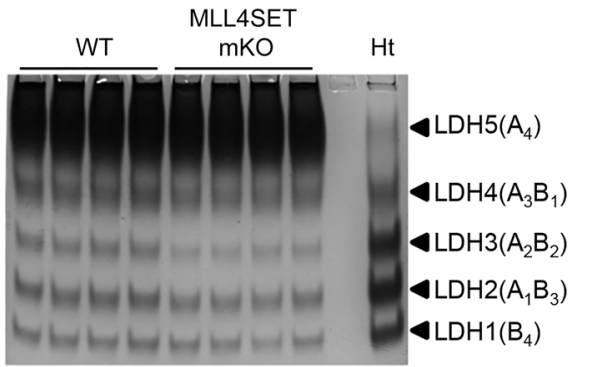

$B_{G O}$

$\begin{array}{lcc} & & \\ \text { co cellular compartments term } & \text { Count } & P \text {-value } \\ \text { cytoplasm } & 390 & 8.20 \mathrm{E}-10 \\ \text { membrane } & 401 & 1.30 \mathrm{E}-08 \\ \text { myofibril } & 11 & 9.30 \mathrm{E}-06 \\ \text { sarcoplasmic reticulum } & 11 & 2.70 \mathrm{E}-04 \\ \text { sarcolemma } & 16 & 3.20 \mathrm{E}-04 \\ \text { myosin complex } & 10 & 5.30 \mathrm{E}-04 \\ \text { I band } & 7 & 6.00 \mathrm{E}-04 \\ \text { cell surface } & 47 & 1.20 \mathrm{E}-03 \\ \text { MHC class II protein complex } & 5 & 1.60 \mathrm{E}-03 \\ \text { neuronal cell body } & 40 & 2.80 \mathrm{E}-03 \\ \text { voltage-gated potassium channel } & 11 & 3.20 \mathrm{E}-03 \\ \text { complex } & 7 & 3.50 \mathrm{E}-03 \\ \text { sarcoplasmic reticulum membrane } & 4 & 4.50 \mathrm{E}-03 \\ \text { troponin complex } & & \end{array}$

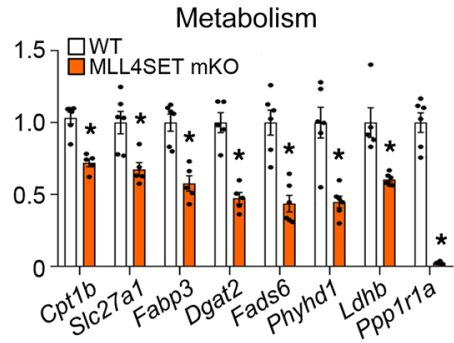

C
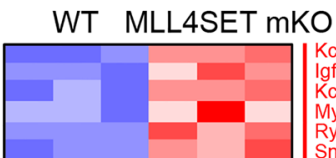
Kcnj3
Igfn1
Kcne11
Myh8
Ryr3 Myh8
Ryr3
Sntb1 Adra1a Myl4 Myh3 Ankrd1 Actc1 Kenc3 Myo1f Fbp2 Sorbs? Thbs4 Tnnt2 Fas Fas Agp4
Pde4d Kane4 Kcne4
Adrb2 Adrb2
Itga7 Trim63 Mylpf Camk2g Sgcb Lmod 1 Pak1 Ankrd2 KIhl40 Cacna1a
Fxyd1,Fxyd7 Fxyd1,Fxy
Cntnap2 Cntnap2
Nos1 Kenc1 Sgca
Casq2 Gstm7 Dhrs7c Myoz2 Cacna1s Myh13 Abra Dmpk
Kcnq4
Scn1b Scn1b Esr1 Pld2 Ablim2 Cd59b Kanf1 Keng4 Mybph Atp2a2 Csrp3 Cacna2d Cacnb1 Kanh2 Cib2 Tnni1 Kcnb1
Smtnl1
Myl2 Myl2
Myh7 Tnnc1
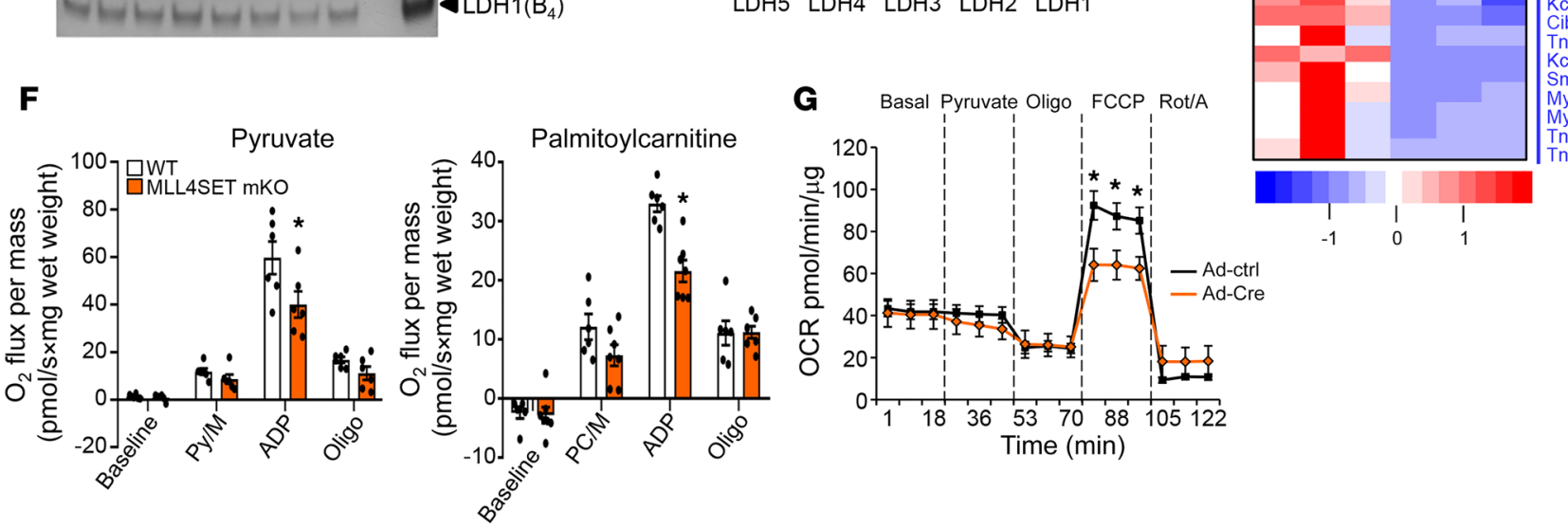

Figure 3. MLL4 coordinately regulates gene programs controlling muscle contraction and energy metabolism. (A) Volcano plot showing fold changes versus $P$ values for analyzed RNA-Seq data generated from the GC muscle of 8-week-old male MII4SET-mKO mice compared with littermate controls (WT). Significantly upregulated genes are represented by red dots, whereas downregulated genes are represented by blue dots. (B) GO enrichment analysis of gene transcripts regulated in MIILSET-mKO muscle. (C) Heatmap analysis of contraction-related genes regulated in MIILSET-mKO muscle compared with WT controls. $n=3$ independent samples per group. Color scheme for fold change is provided. (D) Expression of genes (qRT-PCR) involved in muscle contraction, calcium handling, and metabolism in GC muscle from the indicated genotypes. $n=5-6$ mice per group. (E) Left: LDH isoenzymes were separated by polyacrylamide gel electrophoresis using whole-cell extracts from heart ( $\mathrm{Ht}$, control) and GC muscle from indicated mice. A representative gel showing 4 independent mice per group is shown. Right: quantification of LDH isoenzyme activity gel electrophoresis shown on the left. (F) Mitochondrial respiration rates were determined from the EDL muscle of the indicated genotypes using pyruvate or palmitoylcarnitine as a substrate. Pyruvate/malate (Py/M) or palmitoylcarnitine/malate (PC/M) stimulated, ADP-dependent respiration, and oligomycin induced (oligo) are shown. $n=6-7$ mice per group. (G) OCRs in skeletal myotubes harvested from MII4SET ${ }^{f / f l}$ mice subjected to adenovirus-based overexpression of Cre compared with control virus. Basal OCR was first measured, followed by administration of $10 \mathrm{mM}$ sodium pyruvate, and $2 \mu \mathrm{M}$ oligomycin (to inhibit ATP synthase), uncoupler FCCP ( $2 \mu \mathrm{M})$, or rotenone/ antimycin (Rot/A; $1 \mu \mathrm{M}$ ), as indicated. $n=6$ separate experiments done with 5 biological replicates. Values are represented as mean \pm SEM. ${ }^{*} P<0.05$ vs. corresponding controls, 2-tailed unpaired Student's $t$ test. 


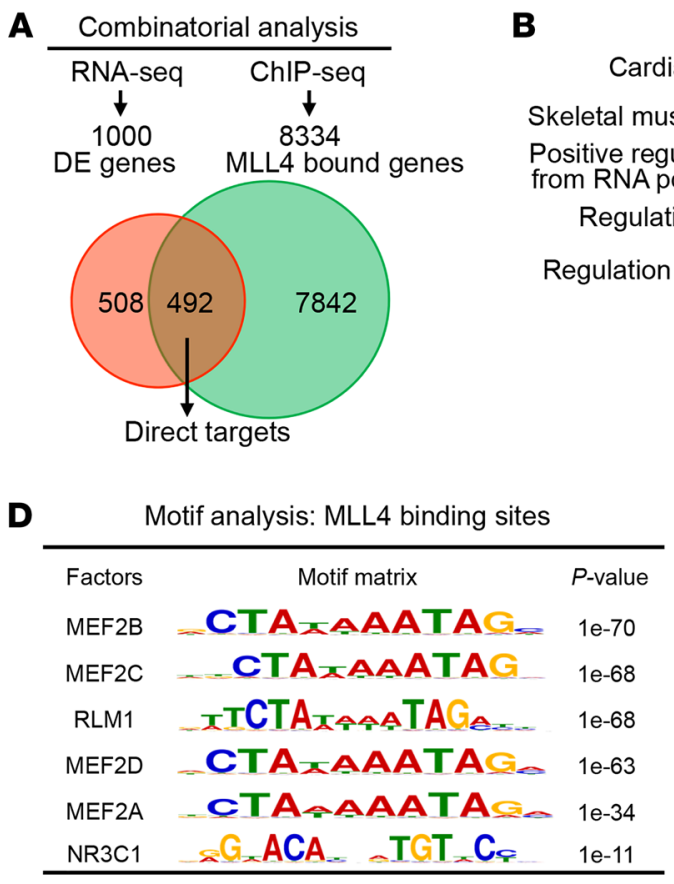

$\mathbf{F}$

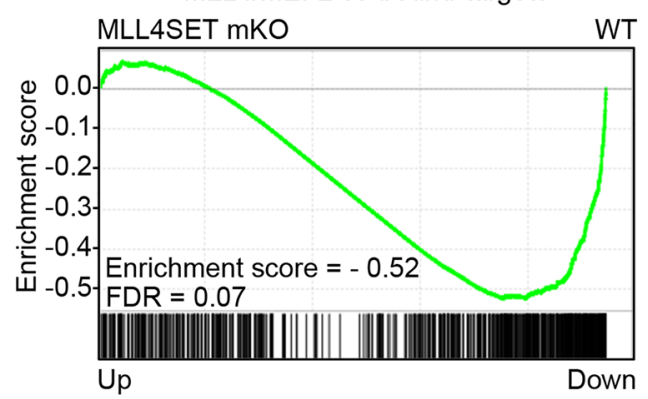

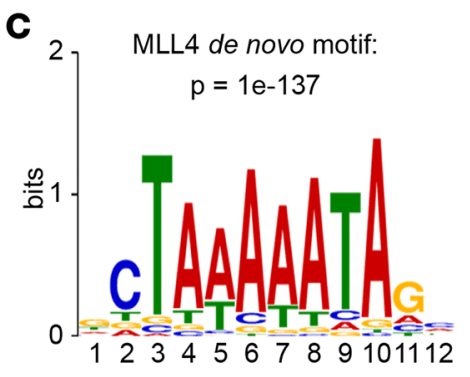
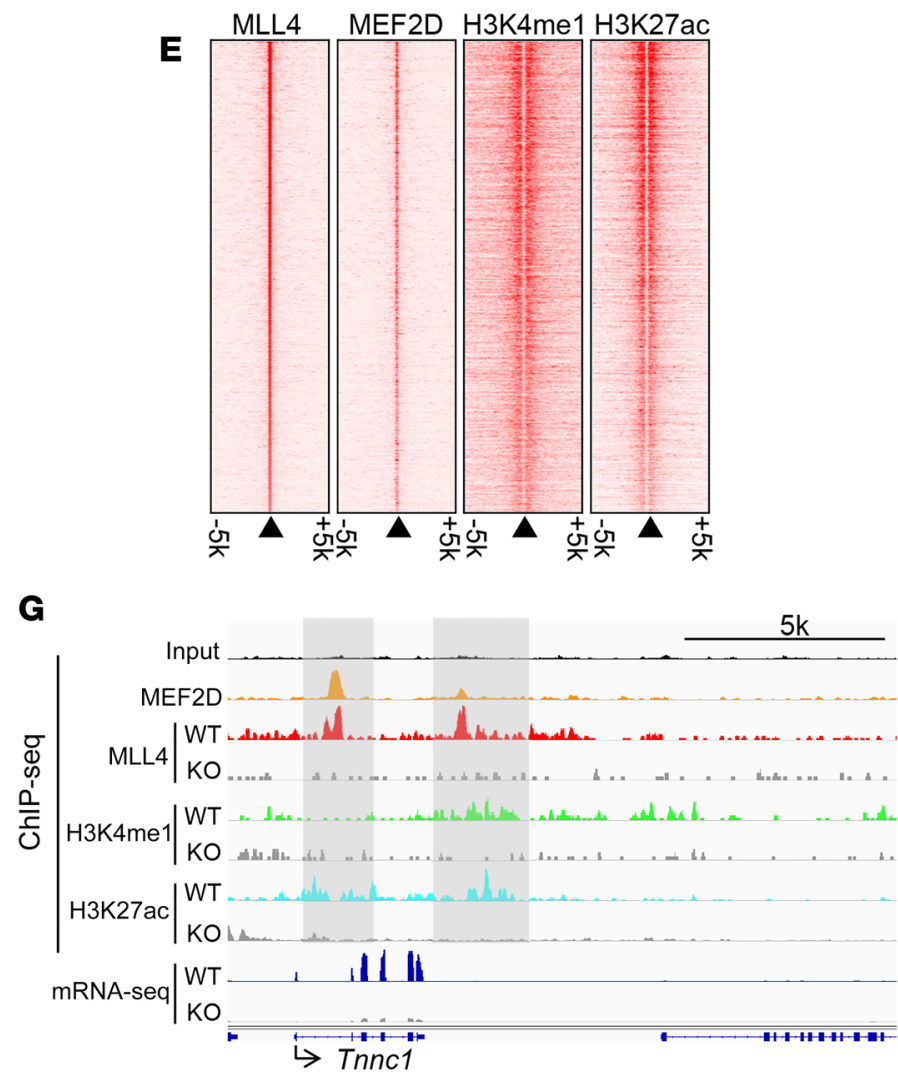

H

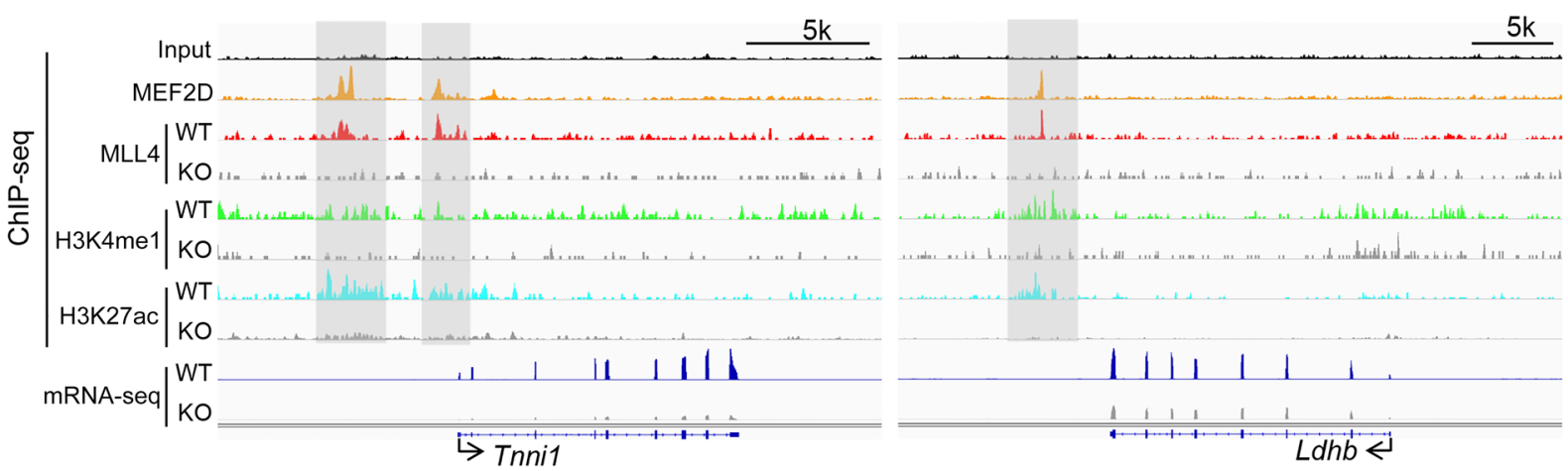


Figure 4. MLL4 colocalizes with MEF2 on enhancers of slow oxidative muscle genes. (A) Analysis of the MLL4 ChIP-Seq data in muscle and myocytes combined with mRNA-Seq data set upon muscle M/l4 deletion defines a set of genes directly regulated by MLL4. (B) GO enrichment analysis of MLL4 direct targets, with the top 5 terms shown. (C) De novo motif analysis of MLL4-binding regions in muscle using HOMER. Shown is the top-scoring motif present in the top 5000 emergent MLL4-binding sites. (D) Known TF motifs with the highest relative enrichment in MLL4-binding regions in muscle. (E) MLL4 colocalizes with MEF2 on active enhancers in myocyte. Heatmap shows the ChIP-Seq binding signal intensity for MLL4, MEF2D, H3K4me1, and H3K27ac. Binding is ranked from the strongest to the weakest MLL4-binding sites. Active enhancers are defined with enhancer markers H3K4me1 and H3K27ac. (F) GSEA showing that expression of MLL4/MEF2 cobound target genes is significantly downregulated in MII4SET-mKO muscle compared with that of WT controls. All genes in mRNA-Seq profiling from MII4SET-mKO muscle were ranked by fold difference compared with WT controls and expressed on the $x$ axis. This data set was compared with the gene list of the nearest genes identified in MLL4/MEF2 cobound regions in muscle. (G and $\mathbf{H}$ ) MLL4-dependent active enhancers on slow oxidative gene loci are shown. Top: ChIP-Seq binding profiles for MLL4, MEF2D, and histone modifications in WT or MII4-KO myocytes. mRNA-Seq data from WT and MII4SET-mKO muscle is shown at the bottom, indicating a high correlation of the 2 data sets. Input, genomic DNA from myocytes. Gray boxes indicate the high-confidence MLL4binding regions corresponding to Tnnc1, Tnni1, and Ldhb genes. Data were obtained from published data sets CSE50466 and GSE43223. The current data sets CSE138994 and CSE137368 were also analyzed.

enriched with muscle contraction-related terms similarly to those seen in the Mll4SET-mKO transcriptome (Figure 4B). In addition, we also found that MLL4 could occupy many fatty acid utilization genes (e.g., Cpt1b and Fabp3) (Supplemental Figure 10E). Together, the strong correlation between the cistromic and transcriptomic findings supports a direct role for MLL4 in regulating muscle fiber-type gene programs.

Interestingly, de novo motif analysis of the top 5000 emergent MLL4-binding regions in muscle using HOMER revealed a sequence element, $5^{\prime}$-CTAAAAATAG-3', as the highest score motif, with a $P$ value of $1 \times 10^{-137}$ (Figure $4 \mathrm{C}$ ). This motif closely corresponded to a consensus site that has been previously known as the MEF2-binding site. Indeed, the search for known motifs confirmed that the most enriched motif within MLL4-binding regions was the binding site for MEF2 family members, key regulators of the type I fiber-type program (refs. 18, 39, and Figure 4D). Moreover, we found that MLL4 could occupy both Mef $2 a$ and $M e f 2 d$ genes (Supplemental Figure 10F), indicating that $M e f 2 a$ and Mef $2 d$ are also direct targets of MLL4. These results suggest that MEF2s may be coregulatory transcription factors that are involved in the MLL4-mediated regulation of the type I fiber-type program. We next interrogated a published myocyte MEF2 ChIP-Seq data set (40) in conjunction with our previously published myocyte ChIP-Seq data for MLL4 and histone modifications (H3K4me1 and $\mathrm{H} 3 \mathrm{~K} 27 \mathrm{ac}$ ) associated with enhancer activities (27). As shown in Figure 4E, heatmap visualization of the MEF2 binding and H3K4me1 and H3K27ac signal at the MLL4-binding events demonstrated a substantial degree of MLL4 and MEF2 cooccupancy on active enhancers (Figure $4 \mathrm{E})$. Gene set enrichment analysis (GSEA) further demonstrated that Mll4 deletion significantly downregulated expression of
MLL4 and MEF2 cobound target genes in muscle (Figure 4F). These data suggest that MLL4 acts coordinately with MEF2 in regulating muscle gene expression. Genomic colocalization of MLL4 and MEF2 was confirmed on many slow oxidative muscle fiber gene loci in myocytes. For example, slow fiber-related gene enhancers (e.g., Myh7, Tnnc1, Tnni1, Tnnt1, Ldhb, Casq2, and $M y b p h$ ) showed strong MLL4 and MEF2 binding as well as the presence of H3K4me1 and H3K27ac signals (Figure 4, G and $\mathrm{H}$, and Supplemental Figure 11). Importantly, deletion of Mll4 markedly decreased levels of H3K4me1 and H3K27ac, which was consistent with pronounced reduction of slow oxidative muscle fiber gene mRNA in Mll4SET-mKO muscle (Figure 4, G and $\mathrm{H}$, and Supplemental Figure 11). Together, these results suggest that MLL4 occupies the enhancers in cooperation with MEF2 to activate transcription of the slow oxidative muscle genes.

A series of co-immunoprecipitation (co-IP) studies were next conducted to determine whether MLL4 interacts with MEF2. HEK293 cells were cotransfected with expression vectors for HA-MEF2A and Flag-MLL4. Anti-HA was found to co-IP MLL4 and MEF2A (Figure 5A). Using MLL4 as the IP target, MEF2A was pulled down (Figure 5B). Furthermore, IP of MLL4 in C2C12 myotubes with an anti-MLL4 antibody confirmed that endogenous MLL4 and MEF2 actually interact in muscle cells (Figure 5C). To explore functional correlates of the MLL4/MEF2 interaction, we focused on the $M y h 7$ and $M y h 7 b$ loci. These 2 MLL4regulated genes encode the major slow myosin and drive expression of miR-208b and miR-499, which further promote muscle fiber remodeling $(41,42)$. Upon examination of the MLL4 and MEF2 ChIP-Seq data, we uncovered a MLL4/MEF2 cobound region located approximately $3 \mathrm{~kb}$ upstream of the $M y h 7$ transcription start site (TSS). We identified 2 highly conserved DNA sequences conforming to the consensus binding sites for MEF2 ([C/T]TA[T/A $]_{4} \mathrm{TA}[\mathrm{G} / \mathrm{A}]$ ) in the $M y h 7 / m i R-208 b$ cis-proximal enhancer region (Figure 5D). Cell cotransfection studies were next conducted using a rat $3.5 \mathrm{~kb} M y h 7$ promoter reporter containing the enhancer element (Figure $5 \mathrm{E}$ ). The $M y h 7$ luciferase reporter was not activated by MLL4 alone, but when it was expressed together with MEF2A, synergistic activation was observed (Figure $5 \mathrm{E}$ ), suggesting a cooperative transcriptional activation by the 2 factors. To further assess the activation of the $M y h 7 / m i R-208 b$ enhancer by MLL4/MEF2, deletion mapping studies were used to demonstrate that this MLL4/MEF2 synergistic effect was markedly diminished by deleting the upstream enhancer sequences (Figure 5F). We also detected MLL4 and MEF2 colocalization on the $M y h 7 b$ promoter region (Figure $5 \mathrm{G}$ ). Cell cotransfection studies were further conducted using a mouse $M y h 7 b$ promoterreporter containing a highly conserved MEF2-binding site (Figure $5 \mathrm{H})$. As expected, the combination of MLL4 and MEF2 resulted in synergistic activation of the $M y h 7 b$ reporter (Figure $5 \mathrm{H}$ ). This synergistic effect was completely abolished upon mutation of the MEF2 site (Figure $5 \mathrm{H}$ ), providing further evidence that the MEF2 site cooperates with MLL4 in the activation of $M y h 7 b / m i R-499$ gene transcription. Taken together, these results demonstrate that MLL4 cooperates with MEF2 to activate slow muscle gene transcription, thereby driving fiber-type remodeling.

$M L L 4$ levels are regulated in response to physiological and pathophysiological stimuli in human muscle. To determine the relevance 
A

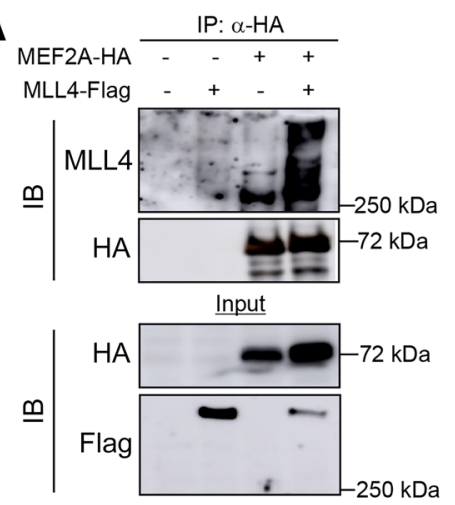

B
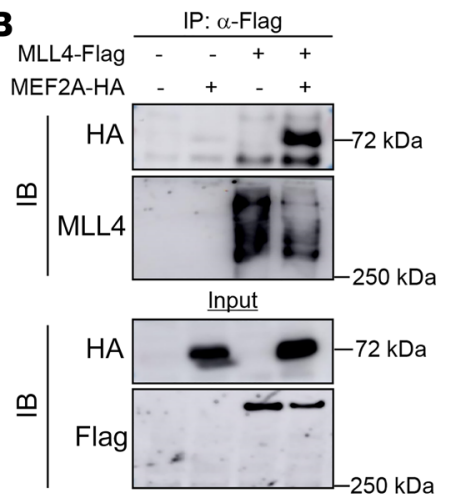

C

IP

$\operatorname{lgG}$ MLL4

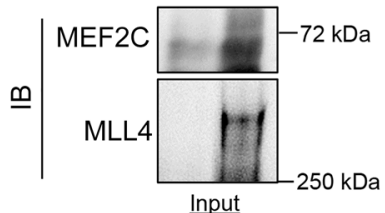

$\left.\cong\right|_{M L L 4} ^{M E F 2 C} \because \underbrace{-72 \mathrm{kDa}}_{250}$
D

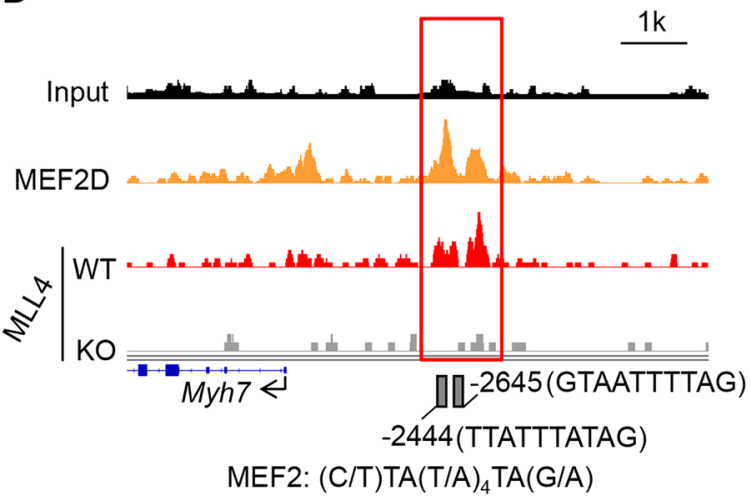

E $\quad=$ MEF2 site

\section{Myh7/miR-208b}
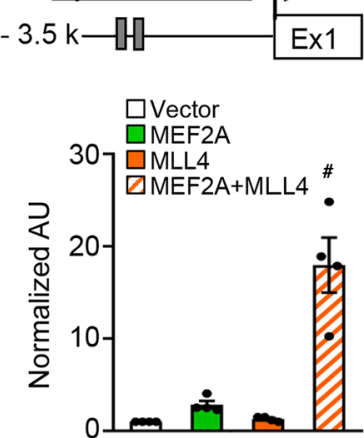

$\mathbf{F}$

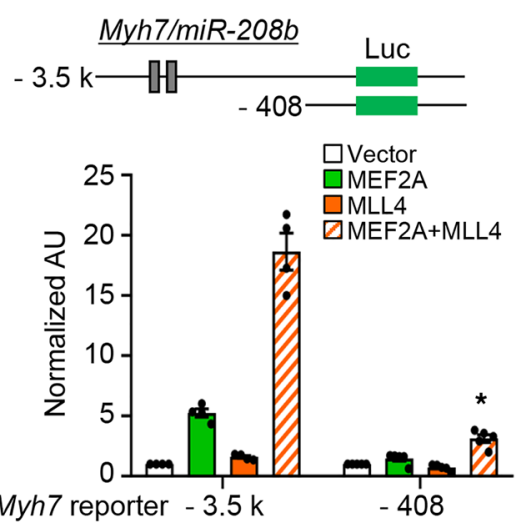

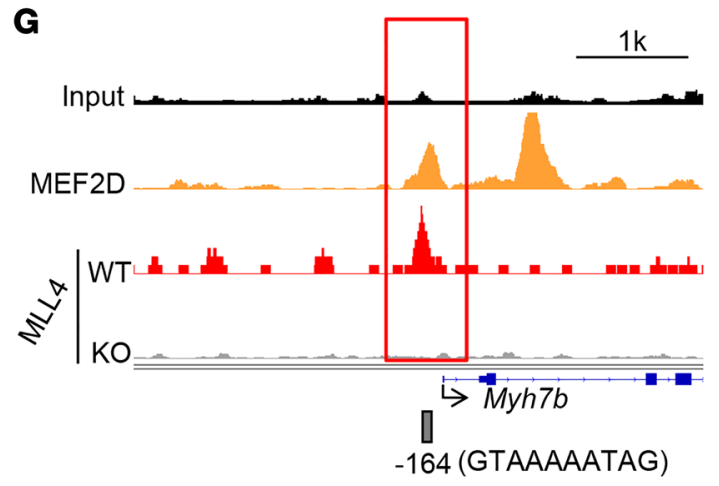

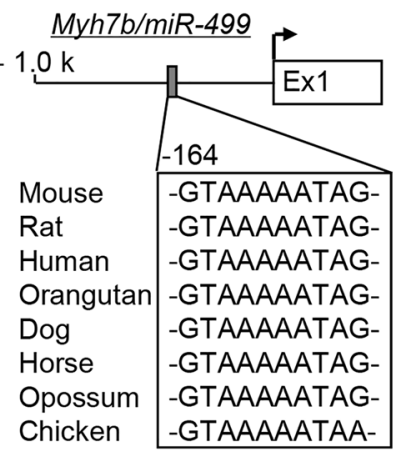

H

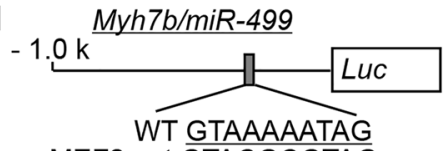

MEF2mut GTACGCGTAG

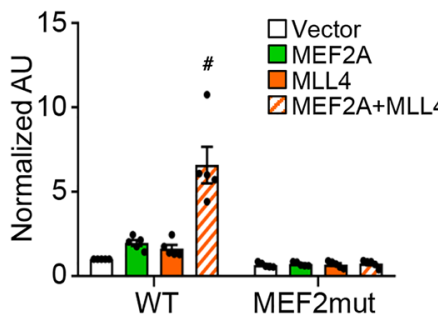

Figure 5. MLL4 cooperates with MEF2 to activate slow myofiber gene transcription. (A and B) Co-IP experiments were performed by cotransfecting HA-MEF2A and Flag-MLL4 in HEK293 cells, as indicated at the top. Antibodies against the HA or Flag epitope were used for co-IP. Extracts (Input) from the HEK293 cells and proteins from the IP were analyzed by immunoblotting. Representative results for co-IP are shown. $n=3$ independent experiments. (C) Co-IP results with extracts prepared from C2C12 myotubes using anti-MLL4 antibody or control IgG. Representative results are shown. $n=3$ independent experiments. (D) MLL4 and MEF2D ChIP-Seq tracks from myocytes at the Myh7 locus. Two putative conserved MEF2-binding sites within the cis-proximal enhancer region of the Myh7 gene are shown. (E) MLL4 and MEF2 synergistically activate Myh7 gene promoter. The rat Myh7.Luc.3.5k promoter reporter was used in cotransfection studies in HEK293 cells in the presence of expression vectors indicated. Values represent mean ( \pm SEM) firefly/renilla luciferase activity shown as arbitrary units (AU) normalized $(=1.0)$ to vector control. $n=4$ independent experiments. (F) Results of transient transfection performed with rMyh7.Luc.3.5k and truncation mutant of rMyh7.Luc.408 in HEK293 cells in the presence of expression vectors indicated. $n=$ 4-5 independent experiments. (C) Left: MLL4 and MEF2D ChIP-Seq tracks from myocyte at the Myh7b locus. Right: putative conserved MEF2-binding site within the mouse Myh7b promoter regions. (H) Top: site-directed mutagenesis was used to abolish the MEF2 response element. Bottom: mMyh7b.Luc.1k (WT) or MEF2mut.mMyh7b.Luc.1k promoter reporters were used in cotransfection studies in HEK293 cells in the presence of expression vectors indicated. $n=5$ independent experiments. Values are represented as mean $\pm \mathrm{SEM} .{ }^{*} P<0.05$ vs. corresponding controls; ${ }^{*} P<0.05$ compared with $M E F 2 A$ alone. $P$ values were determined using 1-way ANOVA coupled to a Fisher's LSD post hoc test.

of the MLL4 regulatory circuit in humans, we investigated whether MLL4 function could be altered by physiological or pathophysiological stimuli in human muscle. We first analyzed the gene expression profiles in human muscle (NCBI's Gene Expression Omnibus database [GEO] GSE1718) from a group of sedentary subjects who underwent 20 weeks of an endurance exercise training program (43). This analysis revealed that the MLL4 regulatory circuit was induced in human muscle by exercise training. Increased mRNA 
levels of $M L L 4$ associated with elevated expression of its targets (MYH7B, TNNT1, MYL2, and ATP2A2) were observed in the muscle tissue from the "posttraining" group compared with the "pretraining” group (Figure 6A). In contrast, the levels of TNNI2 and TNNC2 mRNA showed a decrease in posttraining muscle (Figure 6A).

Skeletal muscle dysfunction, including fiber-type switching and reduced oxidative capacity, has been associated with a variety of human diseases. Patients with adolescent idiopathic scoliosis (AIS), the most common type of scoliosis, which affects $1 \%-4 \%$ adolescents, have generalized muscle dysfunction (44, 45). Although causal mechanisms of AIS remain unclear, paraspinal muscle imbalance due to muscle fiber-type switching has long been recognized as a possible factor underlying the pathology of AIS (46-48). Therefore, we examined the regulation of MLL4 signaling in paraspinal muscle samples from 40 patients with AIS. Consistent with previous reports $(47,48)$, metachromatic ATPase staining revealed that muscle fiber-type programs were altered in paraspinal muscle from AIS patients. The biopsy specimens from the concave side (MV) of the curvature contained a lower percentage of type I muscle fibers compared with the convex side (MX) (Figure 6B). As expected, muscle biopsies from the MV group exhibited lower slow fiber gene (MYH7, TNNI1, TNNC1, TNNT1, and $L D H B$ ) expression, but higher fast fiber gene (TNNI2 and TNNC2) expression compared with those from the MX group (Figure 6C and Supplemental Figure 12A). Expression levels of MLL4 and MEF2s were also lower in the MV group compared with the MX group (Figure 6C). In addition, the levels of MLL4 mRNA exhibited a significant positive correlation with slow fiber gene (MYH7, TNNI1, TNNT1, and LDHB) and MEF2s mRNA levels, but not with a marker of the fast fiber program (TNNI2) (Figure 6D and Supplemental Figure 12B). The relationship between $M L L 4$ expression and levels of the $M E F 2 s$ with type I fiber percentage was also assessed. As shown in Figure 6E, both MLL4 and MEF2s exhibited a significant positive correlation with type I fiber percentage (Figure 6E). Together, these results strongly suggest that the MLL4 regulatory circuit is operative in the regulation of the type I muscle program in response to physiological and pathophysiological stimuli in humans.

\section{Discussion}

The mechanisms underlying the precise orchestration of contractile and metabolic gene expression that specify muscle fiber identity and function remain unclear. Herein, using loss-of-function strategies in mice and primary muscle cells together with assessment of muscle biopsies from humans, we have uncovered an essential role for the enhancer regulator MLL4 in controlling the structural and metabolic programs that govern myofiber identity and muscle performance (Figure 7). MLL4 is highly expressed in type I muscle fibers, and deletion of Mll4 specifically in skeletal muscle resulted in decreased numbers of type I myofibers and diminished mitochondrial respiration, leading to substantial defects in muscle endurance during exercise. These changes resulted from marked downregulation of the slow oxidative muscle gene programs in skeletal muscle lacking MLL4. We found that MLL4 directly binds to enhancers and functions as a coactivator of MEF2 to activate the transcription of slow oxidative myofiber genes, thereby driving the muscle fibe-type remodeling. More- over, our data also established that the MLL4 regulatory circuit is associated with muscle fiber-type switching in humans. Therefore, MLL4 likely represents a previously unrecognized molecular switch that specifies myofiber structural and metabolic identities that govern muscle performance.

Enhancer activation is a critical step for gene activation. While transcriptional regulation of myofiber gene expression through multiple transcription factors, such as nuclear receptor PPARs, ERRs, MEF2, SOX6, and Tbx15, along with coregulators have been established (13-21, 49, 50), it remains unclear how fiber typespecific patterns of gene expression are controlled at the enhancer activation layer. In this study, we showed that the enhancer regulator MLL4 drives the contractile and metabolic specification of type I muscle fibers in both primary skeletal myotubes and skeletal muscle. We also confirmed a marked reduction in active enhancer hallmarks on slow muscle gene enhancers in Mll4-KO myocytes. Notably, deletion of the enzymatic SET domain destabilized the MLL4 protein, thus limiting the study of the role of its enzymatic activity in muscle. It is also worth noting that we used Mck-Cre and HSA-Cre mice for creating mice with the Mll4 deletion specifically in skeletal muscle in this study and that we did not observe a change in muscle differentiation or development upon muscle Mll4 deletion. In the Mll4SET-mKO model using Mck-Cre, which is not fully active until after birth $(37,38)$, we did not observe significant changes in muscle fiber type in Mll4SET-mKO muscle compared with that of WT controls at stage P10. This could reflect muscle fiber-type remodeling during postnatal development. It is possible that other postnatal programs, such as neuronal or hormone signals, are more dominant during the first weeks after birth (5). Remarkably, we found that MLL4 is required for the maintenance of type I fibers in adult muscle. As such, ablation of muscle MLL4 profoundly affects muscle fatigability and exercise performance. Thus, our study has expanded the role of the enhancer regulator MLL4 to include pivotal muscle fiber specification and exercise physiological functions other than development and cell differentiation. Conceivably, this muscle-specific regulatory action may reflect the multifunctions of MLL4 in using its enhancer remodeling activity to direct context-specific biological processes.

Type I muscle fibers are equipped with slow-twitch contractile machinery and a high-capacity fuel-burning system poised for endurance exercise. Our data suggest that MLL4 orchestrates the structural and metabolic programs controlling type I muscle metabolism. Genome-wide transcriptional analysis revealed that MLL4 acts by coordinately regulating genes controlling slowtwitch myofibers, calcium handling, and oxidative metabolism. At a functional level, these changes resulted in substantial impaired muscle endurance during exercise in muscle-specific Mll4-KO mice. Interestingly, we found that muscle MLL4 deficiency did not compromise muscle performance during high-intensity (sprint) exercise, perhaps reflecting that fast type II fibers are involved in rapid bursts of contraction. Our data suggest that MLL4 is a critical upstream epigenetic switch that specifies slow oxidative myofiber identity by coactivating MEF2 transcriptional regulators. Since the expression of slow oxidative muscle genes is also regulated by nuclear receptors, such as PPAR $\beta / \delta$ and ERRs $(14,15$, $17,19)$, it remains to be tested whether MLL4 may also contribute to the activation of PPAR $\beta / \delta$ and ERRs in muscle. Additionally, it 
A

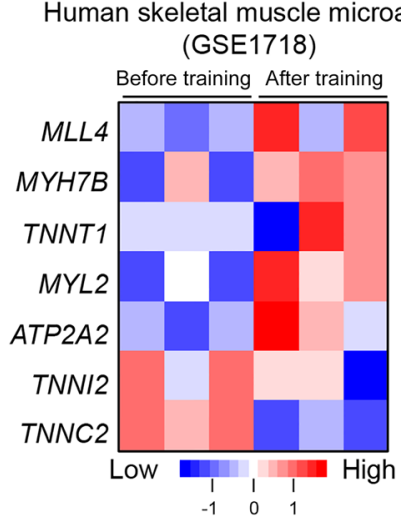

B

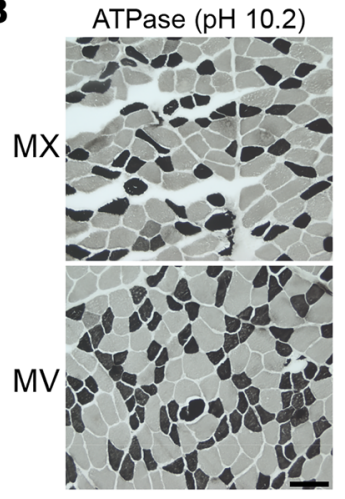

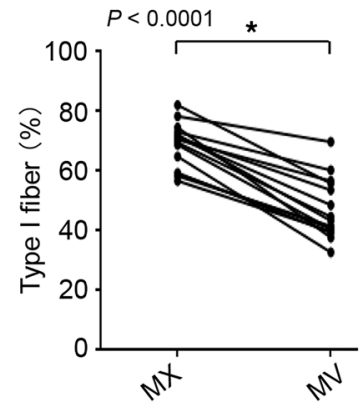
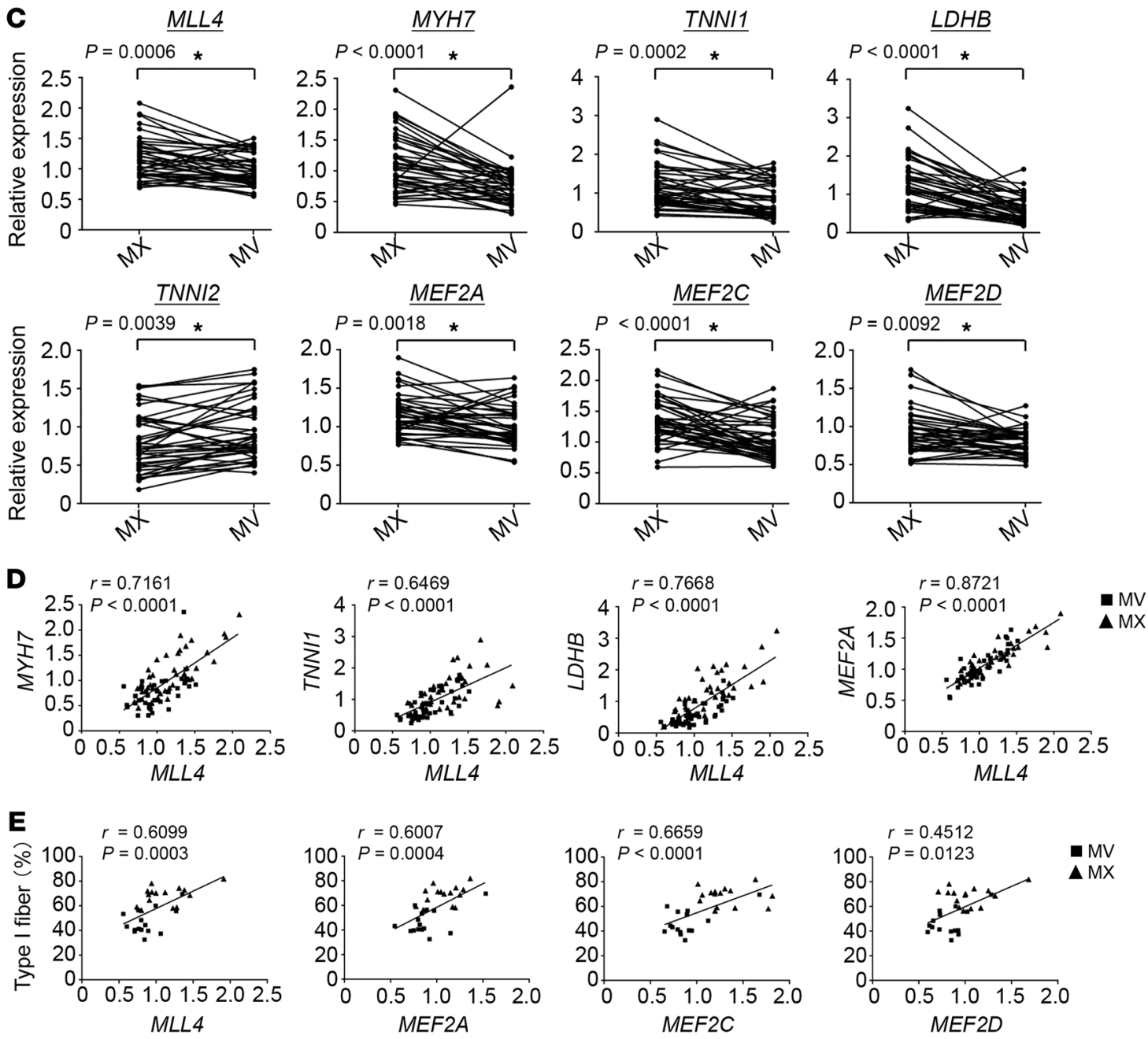

Figure 6. MLL4 regulatory circuit is associated with muscle fiber-type remodeling in humans. (A) Relative expression of $M L L 4$ and representative fiber type-specific genes in human vastus lateralis muscles before or after endurance training. Data were extracted from GEO GSE1718. (B-E) Paraspinal muscle samples from the convex $(M X)$ and concave $(M V)$ sides of the curvature from 40 patients with AIS were used for this analysis. (B) Left: representative sections of paraspinal muscle from AIS patients stained for myosin ATPase activity ( $\mathrm{pH}=10.2$, type II fibers dark, type I fibers light). Scale bar: $100 \mu \mathrm{m}$. Right: quantification of ATPase-staining data shown on the left expressed as mean percentage of total muscle fibers. $n=15$ patients. ${ }^{*} P<0.05$, paired Student's $t$ test. (C) mRNA expression levels of MLL4, MEF2s, and slow oxidative muscle fiber genes were determined by qRT-PCR. $n=40$ patients. ${ }^{*} P<0.05$, paired Student's $t$ test. (D) Correlation between MLL4 gene expression and that of slow oxidative myofiber genes and MEF2s. $n=40$ patients. Pearson's correlation analysis was used to determine the correlation. (E) Correlation between MLL4 and MEF2s expression and the type I fiber percentage. $n=15$ patients. Spearman's correlation analysis was used. 


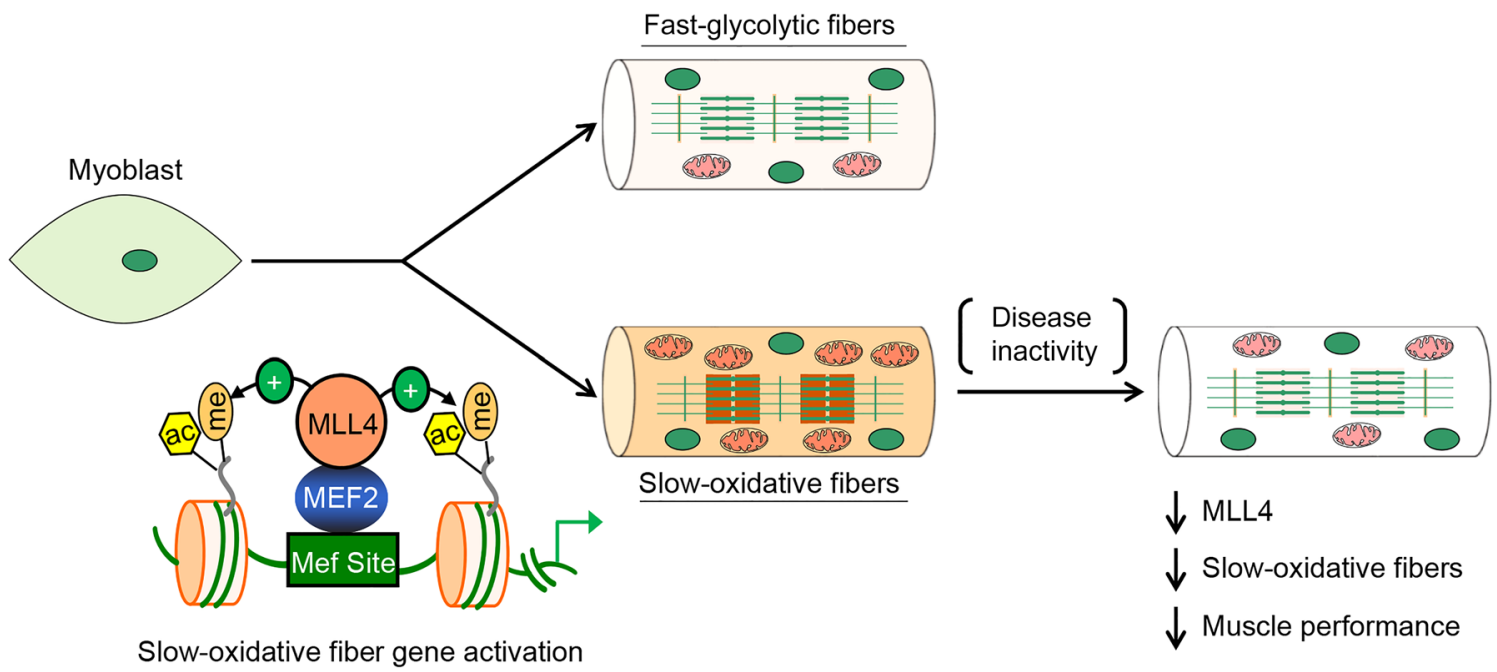

Figure 7. Model of MLL4 in the control of muscle fiber-type specification and function. The schematic depicts a proposed model for the MLL4 regulatory mechanism that specifies muscle fiber identity and muscle performance.

will be of interest to determine whether MLL4 plays an opposing role as a direct transcriptional repressor of fast myofiber-specific gene expression. Coordinate control of muscle fiber type specification and fuel-burning capacity occurs during exercise training. Interestingly, we found that exercise induces an increase in mRNA abundance of MLL4 and its targets in human muscle, suggesting the MLL4 regulatory circuit unveiled here could be involved in the response to exercise training.

Our results from global MLL4-directed transcriptional analysis indicate that the MLL4-driven slow oxidative muscle gene program acts in cooperation with MEF2. The observed role of MEF2 is of interest, given its known role in the regulation of muscle development and muscle fiber-type remodeling in adulthood $(18,39)$. We found that MLL4 interacts and cooperates with MEF2 to activate slow oxidative muscle gene transcription. This is consistent with the observation that elevated expression of MLL4 parallels high MEF2 activity in slow fiber-dominant soleus muscle. Notably, MLL4 also directly binds to Mef $2 a$ and Mef $2 d$ gene loci, which likely adds another regulation layer downstream of MLL4 to enhance the mechanisms described here and boosts the highest level of MLL4/MEF2 cooperation. MEF2D has been shown to recruit the Ash2L methyltransferase complex to MyoD target genes during myogenesis (51). The interaction between MLL4 and MEF2 and the genomic colocalization of MLL4 with MEF2 suggest that MEF2 plays critical roles in recruiting MLL4 to establish active enhancers on slow oxidative muscle genes. The precise mechanism whereby MLL4 transcriptional activation complex is tethered to MEF2-binding sites to establish active enhancers, however, remains to be determined. The class II HDACs and NCoR1 are known to corepress MEF2 (18, 21); therefore, it is likely that MEF2-mediated regulation of the slow fiber phenotype is controlled by the balance between MLL4 and class II HDACs/NCoR1 signaling. MLL4 and MEF2 cooperation allows enhancer activation and the establishment of slow fiber-specific gene expression patterns, while the class II HDACs and NCoR1 put brakes on the activation of MEF2. Interestingly, previous studies also suggest that the muscle-specific switch in exon usage for MEF2 leads to the recruitment of alternate coregulatory complexes $(40,52)$. It is also possible that MEF2 exon switching could alter its association with MLL4.

Myofiber shift from slow oxidative toward fast glycolytic has been associated with a variety of chronic illnesses, including metabolic disorders and muscular diseases $(3,7,12)$. In this study, our survey of the human muscle samples demonstrated that the MLL4 regulatory circuit is associated with muscle fiber-type switching in AIS patients. Specifically, MLL4 and MEF2s levels were reduced in muscle samples from the MV group compared with those in the MX group. We also found that the expression of MLL4 and MEF2s was strongly correlated with the expression of slow fiber genes and type I fiber-type proportion. Based on our data, it is possible that downregulated MLL4 signaling leads to reduced type I muscle fibers, contributing to the paraspinal muscle imbalance in AIS patients. Notably, MLL4 has also been identified as a major causative mutation gene in Kabuki syndrome, a human genetic disease that causes multiple malformations, including muscle hypotonia $(34,53)$. It is tempting to speculate that loss-of-function mutations in MLL4 contribute to the muscle dysfunction in Kabuki syndrome.

In summary, we demonstrate an essential role for enhancer regulator MLL4 in specifying myofiber structural and metabolic identities that govern muscle performance. Given that many disease states are associated with reduced muscle endurance, these findings provide the possibility of therapeutic opportunities for enhancing muscle fitness to combat a variety of metabolic and muscular diseases.

\section{Methods}

Animal studies. Male C57BL/6J WT mice were from MARC of Nanjing University. Generation of $M l l 4^{f l / f l}$ and Mll4SET $T^{f / f l}$ mice has been described elsewhere $(27,29)$. Mice were backcrossed to the C57BL/6J background for more than 6 generations. To generate mice with a muscle-specific disruption of the Mll4 allele, Mll $4^{f / f l}$ and Mll4SET $T^{f / f l}$ mice were crossed with mice expressing Cre recombinase under the control of an Mck promoter (Jackson Laboratory, stock no. 006475) or an HSA promoter (Jackson Laboratory, stock no. 006139) to 
achieve muscle-specific deletion of Mll4. Male offspring were genotyped, and mice at the age of 1 day to 20 weeks were used. Mice were randomly assigned to various analyses. Littermate controls were used in all cases. Investigators involved in immunofluorescence (IF) imaging, RNA-Seq/ChIP-Seq, and histological analysis were blinded. Investigators performing animal handling, sampling, and raw data collection were not blinded.

Human studies. Details on subject characteristics are provided in Supplemental Table 1. AIS patients who underwent posterior instrumentation and spinal fusion surgery were recruited from the Drum Tower Hospital. Patients with scoliosis secondary to known etiology were excluded from the present study. Deep paraspinal muscle biopsies were taken at both the concave and convex sides of the curve's apex during spinal fusion surgery, cleaned, and mounted for fiber typing or flash frozen in liquid nitrogen for RNA isolation.

Exercise stress test. Mice were acclimated (run for 9 minutes at 10 $\mathrm{m} / \mathrm{min}$ followed by 1 minute at $20 \mathrm{~m} / \mathrm{min}$ ) to the treadmill for 2 consecutive days before the experimental protocol. Low -intensity (endurance) exercise studies were conducted as described previously (41, 54). In brief, fed mice were run for 10 minutes at $10 \mathrm{~m} / \mathrm{min}$, followed by a constant speed of $20 \mathrm{~m} / \mathrm{min}$ until exhaustion.

RER during exercise were determined as described previously (41, $54,55)$. Briefly, mice were placed in an enclosed treadmill attached to the Comprehensive Laboratory Animal Monitoring System (CLAMS) (Columbus Instruments) for 10 minutes at a $10^{\circ}$ incline and at $0 \mathrm{~m} /$ min. To determine maximal exercise capacity, mice were subjected to a high-intensity exercise (wind sprints) test consisting of an increasing speed every 2 minutes at $10^{\circ}$ inclination until exhaustion. The increasing speeds used in the protocol were 10, 14, 18, 22, 26, 28, 30, 32, 34, $36,38,40,42,44,46$, and $48 \mathrm{~m} / \mathrm{min}$. One week later, the same group of mice performed a low-intensity exercise (endurance) challenge. After a brief warm-up, the mice were challenged with a constant speed of $60 \%$ of their maximal running speed at $10^{\circ}$ inclination until exhaustion. Measurements were collected before the exercise challenge and throughout the challenge.

Metabolic measurements in vivo. Mice were housed individually in metabolic cages under a 12-hour light/12-hour dark cycle with free access to food and water using the CLAMS (Columbus Instruments). Mice were acclimated in the metabolic cage for 1 day before the recording, according to the manufacturer's instructions. Food, energy expenditure, physical activity, $\mathrm{VO}_{2}$, and $\mathrm{VCO}_{2}$ were assessed simultaneously.

Body composition analyses. Mouse body-composition parameters, including fat mass and lean tissue mass, were determined via dual-energy x-ray absorptiometry (DEXA) using a Lunar PIXImus II Densitometer (GE Healthcare) according to the manufacturer's instructions.

Blood and tissue chemistry. Blood glucose levels were determined using a OneTouch UltraMini glucose meter (OneTouch). Serum TG levels were determined using a Triglyceride Kit (Wako, 290-63701). Serum fatty acid levels were determined using a NEFA Kit (Wako, 294-63601). Serum $\beta$-hydroxybutyrate levels were measured using the $\beta$-Hydroxybutyrate (Ketone Body) Colorimetric Assay Kit (Cayman, 700190) according to the manufacturer's instructions.

Histological analyses. Mouse muscle tissues were frozen in isopentane that had been cooled in liquid nitrogen. IF stains were conducted as previously described (41). For IF stains, the muscle fibers were stained with antibodies directed against MHC1 (BA-D5, catalog AB 2235587; Developmental Studies Hybridoma Bank) or MHC2b (BF-
F3, catalog AB 2266724; Developmental Studies Hybridoma Bank). Tissue sections were stained with H\&E (MilliporeSigma) according to standard protocols. Wheat germ agglutinin (WGA) staining was performed using FITC-conjugated WGA (MilliporeSigma, L4859). Quantification of cross-sectional area of the myofibers was performed with Image J software (NIH).

RNA-Seq studies. Transcriptomics analyses were performed using RNA-Seq as described previously (56). Total RNA was isolated from the GC muscle of 8-week-old male Mll4SET-mKO and WT control mice using RNAiso Plus (Takara Bio). RNA-Seq using Illumina HiSeq 4000 was performed by the Beijing Novogene Bioinformatics Technology Co. Three independent samples per group were analyzed. Paired-end, $150 \mathrm{nt}$ reads were obtained from the same sequencing lane. Transcriptome sequencing libraries averaged 33 million paired reads per sample, with $81.8 \%$ alignment to the mouse genome (UCSC mm10). The sequencing reads were then aligned to the UCSC mm10 genome assembly using TopHat, version 2.0.14, with the default parameters. Fragments per Kb of exon per million mapped reads (FPKM) were calculated using Cufflinks, version 2.2.1. The criteria for a regulated gene were a fold change greater than 1.5 (either direction) and a significant $P$ value $(<0.05)$ versus WT. For pathway analysis, the filtered data sets were uploaded into DAVID Bioinformatics Resources 6.8 to review the biopathways using the Functional Categories database.

The GO analysis was used to interpret data, and the regulated terms ranked by $P$ value are shown in Figure 3B and Figure 4B. The volcano plot and heatmap analysis of regulated genes were generated by using R software, version 3.3.2, and the ggplot2/gplots package. The RNA-Seq data were deposited in the NCBI's GEO database (GSE137368).

RNA analyses. Quantitative reverse-transcriptase PCR (qRT-PCR) was performed as described previously (56). Briefly, total RNA was extracted from mouse muscle or primary myotubes using RNAiso Plus (Takara Bio). Isolated total RNA integrity was electrophoretically verified by ethidium bromide staining. Then, $1 \mu \mathrm{g}$ total RNA samples were reverse transcribed with the PrimeScript RT Reagent Kit with gDNA Eraser (Takara Bio) using random hexamer primers according to the manufacturer's instructions. Real-time qPCR was performed using the ABI Prism Step-One System with SYBR Premix Ex Taq (Takara Bio). Specific oligonucleotide primers for target gene sequences are listed in Supplemental Table 2. Arbitrary units of target mRNA were corrected to the expression of $36 b 4$ or GAPDH.

ChIP and ChIP-Seq. ChIP assays from WT or Mll4-mKO mice or primary skeletal myocyte were conducted as described previously, with modifications (56). Briefly, muscle cell chromatin fragmentation was performed by sonication using a Bioruptor (Diagenode). An aliquot of chromatin was precleared with protein $G$ and immunoprecipitated with anti-MLL4, anti-H3K4me1 (Abcam, catalog ab8895), or IgG control (Beyotime, catalog A7016) antibodies. Following reversal of crosslinking, DNA was isolated using the standard phenol-chloroform method. qPCR products were assessed and measured using the ABI Prism Step-One System. Quantitative analysis was performed by the standard curve method. Specific oligonucleotide primers for target regions are listed in Supplemental Table 2. For ChIP-Seq, the MLL4-precipitated DNA samples from 3 independent ChIP experiments were pooled ( $n=3$ mice each WT or Mll4-mKO group) and then amplified according to the ChIP Sequencing Sample Preparation Guide provided by Illumina. The DNA library was generated using the NEBNext Ultra II DNA Library Prep Kit (NEB, E7645). Deep sequenc- 
ing using Illumina HiSeq 2500 was performed by the Beijing Novogene Bioinformatics Technology Co.

ChIP-Seq data processing. ChIP-Seq data analysis was performed as described previously with modifications (56). Briefly, single-end $50 \mathrm{nt}$ reads were mapped to the mouse genome (UCSC mm10) using Bowtie2, version 2.2.5. Only the sequences uniquely mapped with no more than 1 mismatch were kept and used as valid reads. The peak caller program MACS2, version 2.1.1, was used to identify peaks, with the following parameter settings: -keep-dup $=1,-B,-$ SPMR to generate signal pileup tracks in bedGraph format on a per million reads basis. This allowed for direct comparison between the WT and KO ChIP-Seq. Genome regions were associated to the gene with the nearest TSS from the UCSC genome browser. Region centers that were 1 $\mathrm{kb}$ upstream to $200 \mathrm{bp}$ downstream of a RefGene TSS were classified as promoter regions. The remaining region centers were classified based on overlapping RefGene introns, exon coding regions, 5' UTRs, and 3' UTRs. All other regions were classified as intergenic. A total of 8334 MLL4 bound genes in skeletal muscle or myocyte and the global mRNA changes (1000 genes) upon muscle Mll4 deletion were used for the combinatorial analysis to define a set of genes directly regulated by MLL4. GO term enrichment analysis was performed with MLL4 direct targets (492 genes) as input to DAVID. De novo motif discovery of MLL4 ChIP-seq peaks was performed using the findMotifsGenome command in HOMER (-size = given) with default options. The known motif enrichment analysis was performed using the TRANSFAC database and the findMotifsGenome command in HOMER with the following parameter settings: -size = given, - nomotif. $P$ values were calculated on the basis of a binomial test against a GC\%-matched background. We also interrogated a public myocyte MEF2 ChIP-Seq data set (GEO GSE43223) (40) in conjunction with our previously published myocytes ChIP-Seq data for MLL4 and histone modifications (H3K4me1 and H3K27ac) associated with enhancer activities (GEO GSE50466) (27). The colocalization was defined as 2 regions overlapping for at least $1 \mathrm{bp}$, and BEDTools was used for genomic region handling. For heatmaps, tag density matrices were calculated using HOMER followed by visualization by Treeview, version 1.1.6r4. Around $5 \mathrm{~kb}$ centered on each peak with $10 \mathrm{bp}$ bin size was used for MLL4, MEF2D, H3K4me1, and H3K27ac ChIP-Seq data sets. The heatmap color scale indicates the binding signals per million total reads. GSEA was carried out by comparing a data set of MLL4/ MEF2D cobound targets (627 genes) with that from genome-wide mRNA expression profiling upon muscle Mll4 deletion. Enrichment score was obtained with an FDR $q$ value by running sum of the ChIPSeq genes and differentially expressed rank-ordered genes in mRNA profiling. Genome browser tracks of ChIP-Seq data were visualized in IGV, version 2.3.70. ChIP-Seq data were deposited in the NCBI GEO database (GSE138994).

Antibodies and immunoblotting studies. Antibodies were as follows: MHC1 (BA-D5, catalog AB 2235587) and MHC2b (BF-F3, catalog AB 2266724), Developmental Studies Hybridoma Bank; anti-SUZ12 (catalog sc-46264, 1:1,000 dilution), Santa Cruz Biotechnology Inc.; anti-EZH2 ( catalog 5246S, 1:1,000 dilution), Cell Signaling Technology; anti- $\alpha$-tubulin (catalog bs1699, 1:5,000 dilution), Bioworld; anti-MEF2C (catalog ab211493, 1:500 dilution), Abcam; anti-myosin slow (catalog M8421, 1:250 dilution), anti-myosin fast ( catalog M4276, 1:250 dilution), anti-Flag ( catalog F1804, 1:1,000 dilution), and anti-HA ( catalog H9658, 1:1,000 dilution), MilliporeSigma; and
anti-MLL4 (1:2,000 dilution), provided by Kai Ge at NIH (Bethesda, Maryland, USA), as previously described (27). Western blotting studies were performed as previously described (56).

LDH isoenzyme analysis. LDH isoenzyme patterns were determined as previously described (55). Protein extracted from mouse hearts served as the positive control.

Mitochondrial respiration studies. Mitochondrial respiration rates were measured in saponin-permeabilized EDL muscle fibers with pyruvate or palmitoylcarnitine as a substrate, as described previously $(41,55)$. In brief, muscle fibers were separated and transferred to BIOPS buffer (7.23 mM K ${ }_{2}$ EGTA, $2.77 \mathrm{mM} \mathrm{CaK}_{2}$ EGTA, 20 mM imidazole, $20 \mathrm{mM}$ taurine, $50 \mathrm{mM}$ potassium 2-[N-morpholino]-ethanesulfonic acid, $0.5 \mathrm{mM}$ dithiothreitol, $6.56 \mathrm{mM} \mathrm{MgCl}_{2}, 5.7 \mathrm{mM}$ ATP, and $14.3 \mathrm{mM}$ phosphocreatine [PCr], $\mathrm{pH}$ 7.1). The muscle fiber bundles were then permeabilized with $50 \mu \mathrm{g} / \mathrm{mL}$ saponin in BIOPS solution. Measurement of oxygen consumption in permeabilized muscle fibers was performed in buffer $\mathrm{Z}$ (105 mM potassium 2-[N-morpholino]-ethanesulfonic acid, $30 \mathrm{mM} \mathrm{KCl}, 10 \mathrm{mM} \mathrm{KH}_{2} \mathrm{PO}_{4}, 5 \mathrm{mM} \mathrm{MgCl}_{2}, 5 \mathrm{mg} /$ $\mathrm{mL} \mathrm{BSA}, 1 \mathrm{mM}$ EGTA, $\mathrm{pH} 7.4)$ at $37^{\circ} \mathrm{C}$ and in the oxygen concentration range 220 to $150 \mathrm{nmol} \mathrm{O}_{2} / \mathrm{mL}$ in the respiration chambers of an Oxygraph $2 \mathrm{~K}$ (Oroboros Inc.). Following measurement of basal, pyruvate $(10 \mathrm{mM}) /$ malate $(5 \mathrm{mM})$, or palmitoylcarnitine $(40 \mu \mathrm{M}) /$ malate $(5 \mathrm{mM})$ respiration, maximal (ADP stimulated) respiration was determined by exposing the mitochondria to $4 \mathrm{mM}$ ADP. Uncoupled respiration was evaluated following the addition of oligomycin $(1 \mu \mathrm{g} / \mathrm{mL})$. Respiration rates were determined and normalized to tissue wet weight using DATLAB 5 software (Oroboros Inc.), and data were expressed as pmol $\mathrm{O}_{2} \mathrm{~s}^{-1} \mathrm{mg}$ wet weight $\mathrm{t}^{-1}$.

Oxygen consumption measurements. Cellular OCRs were measured using the XF24 analyzer (Seahorse Bioscience Inc.) per the manufacturer's protocol, as described previously (41). Basal OCR was first measured in XF Assay Media without sodium pyruvate, followed by administration of sodium pyruvate to a final concentration of $10 \mathrm{mM}$. Uncoupled respiration was evaluated following the addition of oligomycin $(2 \mu \mathrm{M})$ to inhibit ATP synthase and the uncoupler FCCP $(2 \mu \mathrm{M})$, which was followed by the addition of rotenone/antimycin $(1 \mu \mathrm{M})$. Immediately after measurement, total protein levels were measured with the Micro BCA Protein Assay Kit (Thermo Scientific) for data correction.

Cell transfection and luciferase reporter assays. pCMV6-flag-myc$M L L 4$, rat 3.5-kb $M y h 7$, rat $408 M y h 7$, and mouse $1.0 \mathrm{~kb} M y h 7 b$ promoter reporters have been described previously $(15,57,58)$. The $M E F 2 A$ plasmid was generated by PCR amplification from cDNA of human MEF2A, followed by cloning into the pcDNA3.1 vector. Sitedirected mutagenesis was performed using the QuikChange Kit (Stratagene) according to the manufacturer's protocol: 5'-GTACGCGTAG (Myh7b MEF2mut) (mutated nucleotides are shown in bold). All constructs were confirmed by DNA-Seq. HEK293T cells were obtained from ATCC and were cultured at $37^{\circ} \mathrm{C}$ and $5 \% \mathrm{CO}_{2}$ in DMEM supplemented with $10 \%$ FCS, $1000 \mathrm{U} / \mathrm{mL}$ penicillin, and $100 \mathrm{mg} / \mathrm{mL}$ streptomycin. Transient transfections in HEK293T cells were performed using PEI Transfection Reagent (Polysciences) following the manufacturer's protocol. Luciferase reporter assays were performed as previously described (56). Briefly, $100 \mathrm{ng}$ of reporter was cotransfected with 50 ng of pcDNA3.1-MEF2A and 100 ng of pCMV6-MLL4 and 25 ng of CMV promoter-driven Renilla luciferase to control for transfection efficiency. Cells were harvested 48 hours after transfection. Luciferase assay was performed using Dual-Glo (Promega) according to the 
manufacturer's recommendations. All transfection data are presented as mean \pm SEM for at least 3 separate transfection experiments.

Cell culture and adenoviral infection. Primary muscle cells were isolated from skeletal muscles as previously described (14). For differentiation, cells were washed with PBS and refed with $2 \%$ horse serum/DMEM differentiation medium daily. Primary myoblasts were infected with an adenovirus overexpressing Cre or control virus as previously described (14); 12 hours after infection, cells were induced to differentiate into myotubes for 60 hours before harvest.

IP. Whole lysate from C2C12 myotubes or lysate from HEK293T cells 48 hours after transfection were used for co-IP studies.

HEK293T and C2C12 cells were obtained from ATCC and were cultured at $37^{\circ} \mathrm{C}$ and $5 \% \mathrm{CO}_{2}$ in DMEM supplemented with $10 \% \mathrm{FBS}$, $1000 \mathrm{U} / \mathrm{mL}$ penicillin, and $100 \mathrm{~g} / \mathrm{mL}$ streptomycin. For myocyte differentiation, $\mathrm{C} 2 \mathrm{C} 12$ cells were cultured with $2 \%$ horse serum/DMEM differentiation medium. C2C12 myotubes or HEK293 T cells were collected in lysis buffer (50 mM Tris, pH 7.5, $150 \mathrm{mM} \mathrm{NaCl}, 2 \mathrm{mM}$ EDTA, $1.5 \%$ NP40, $1 \times$ cOmplete [Roche], and $1 \mathrm{mM}$ PMSF), and $1 \mu \mathrm{g}$ of M2 anti-FLAG (MilliporeSigma) or anti-HA (MilliporeSigma) antibodies were incubated with extract and protein $\mathrm{G}$-conjugated agarose beads. The immunoprecipitated proteins were analyzed by immunoblotting.

Statistics. All mouse and cell studies were analyzed by 2-tailed Student's $t$ test or ANOVA coupled to a Fisher's least significant difference (LSD) post hoc test when more than 2 groups were compared. No statistical methods were used to predetermine sample sizes, and sample sizes (ranging from $n=3$ to $n=13$ ) are explicitly stated in the figure legends. All data points were used in statistical analyses. Data represent the mean \pm SEM, with a statistically significant difference defined as a value of $P<0.05$. Statistical analyses in human studies were performed using GraphPad Prism 6 software. Gene expression levels in human studies were analyzed using paired Student's $t$ test, Pearson's correlation test, or Spearman's correlation test. Significant differences were defined as $P<0.05$.

Study approval. All animal studies were conducted in strict accordance with the institutional guidelines for the humane treatment of animals and were approved by the IACUC committees at the MARC of Nanjing University. Human muscle samples were collected from 40 female AIS patients. Clinical ethical approval in compliance with the Declaration of Helsinki was obtained from the Ethical Committee of Drum Tower Hospital. Details of the research project and biopsy collection were explained to all subjects and/or their parents or guardians before entering the study. Informed consent was obtained from all subjects.

\section{Author contributions}

LL and CD performed most of the experiments with assistance from TF, ZF, JEL, L Xiao, ZX, YY, QG, ZS, WS, YM, LY, Z Zhou, $\mathrm{DZ}$, and $\mathrm{L} \mathrm{Xu}$. LL, CD, and ZG designed experiments, discussed data, and wrote the manuscript. Z Zhu, YG, and KG contributed reagents and provided scientific insight and discussion. ZG supervised the work. All authors reviewed and contributed to the manuscript. The order of the co-first authors is based on the length of time spent on the project.

\section{Acknowledgments}

We express special thanks to Daniel P. Kelly (University of Pennsylvania), Aibin He (Peking University), Yong Liu (Wuhan University), and Zhongzhou Yang and Min-Sheng Zhu (Nanjing University) for insightful discussions; and Hao Yin (Shanghai Institute for Biological Sciences) for advice on myotube IF staining studies. This work was supported by grants from the Ministry of Science and Technology of China (National Key R\&D Program of China 2018YFA0800700 and 973 Program 2015CB856300), the National Natural Science Foundation of China (31922033, 91857105 and 31771291), the Natural Science Foundation of Jiangsu Province (BK20170014 and SWYY-002 to ZG), and Fundamental Research Funds for the Central Universities (090314380036 to TF; 090314380031 and 090314380035 to ZG).

Address correspondence to: Zhenji Gan, Model Animal Research Center of Nanjing University, 12 Xuefu Road, Pukou, Nanjing, China 210061. Phone: 86.25.58641546; Email: ganzj@nju.edu.cn.
1. Booth FW, Thomason DB. Molecular and cellular adaptation of muscle in response to exercise: perspectives of various models. Physiol Rev. 1991;71(2):541-585.

2. Yan Z, Okutsu M, Akhtar YN, Lira VA. Regulation of exercise-induced fiber type transformation, mitochondrial biogenesis, and angiogenesis in skeletal muscle. J Appl Physiol. 2011;110(1):264-274.

3. Zierath JR, Hawley JA. Skeletal muscle fiber type: influence on contractile and metabolic properties. PLoS Biol. 2004;2(10):e348.

4. Pette D, Staron RS. Myosin isoforms, muscle fiber types, and transitions. Microsc Res Tech. 2000;50(6):500-509.

5. Schiaffino S, Reggiani C. Fiber types in mammalian skeletal muscles. Physiol Rev. 2011;91(4):1447-1531.

6. Baldwin KM, Haddad F. Effects of different activity and inactivity paradigms on myosin heavy chain gene expression in striated muscle. JAppl Physiol. 2001;90(1):345-357.

7. Bassel-Duby R, Olson EN. Signaling pathways in skeletal muscle remodeling. Annu Rev Biochem. 2006;75:19-37.

8. Holloszy JO, Coyle EF. Adaptations of skeletal muscle to endurance exercise and their metabolic consequences. J Appl Physiol Respir Environ Exerc Physiol. 1984;56(4):831-838.

9. Neufer PD, et al. Understanding the cellular and molecular mechanisms of physical activityinduced health benefits. Cell Metab. 2015;22(1):4-11.

10. Saltin B, Henriksson J, Nygaard E, Andersen P, Jansson E. Fiber types and metabolic potentials of skeletal muscles in sedentary man and endurance runners. Ann N Y Acad Sci. 1977;301:3-29.

11. He J, Watkins S, Kelley DE. Skeletal muscle lipid content and oxidative enzyme activity in relation to muscle fiber type in type 2 diabetes and obesity. Diabetes. 2001;50(4):817-823.

12. Hickey MS, et al. Skeletal muscle fiber composition is related to adiposity and in vitro glucose transport rate in humans. Am J Physiol. 1995;268(3 Pt 1):E453-E457.

13. Arany Z, et al. The transcriptional coactivator
PGC-1beta drives the formation of oxidative type IIX fibers in skeletal muscle. Cell Metab. 2007;5(1):35-46.

14. Gan $Z$, et al. The nuclear receptor PPAR $\beta / \delta$ programs muscle glucose metabolism in cooperation with AMPK and MEF2. Genes Dev. 2011;25(24):2619-2630.

15. Gan Z, et al. Nuclear receptor/microRNA circuitry links muscle fiber type to energy metabolism. J Clin Invest. 2013;123(6):2564-2575.

16. Lin J, et al. Transcriptional co-activator PGC-1 alpha drives the formation of slow-twitch muscle fibres. Nature. 2002;418(6899):797-801.

17. Narkar VA, et al. Exercise and PGC-1 $\alpha$-independent synchronization of type I muscle metabolism and vasculature by ERR $\gamma$. Cell Metab. 2011;13(3):283-293.

18. Potthoff MJ, et al. Histone deacetylase degradation and MEF2 activation promote the formation of slow-twitch myofibers. J Clin Invest. 2007;117(9):2459-2467.

19. Rangwala SM, et al. Estrogen-related receptor gamma is a key regulator of muscle mitochon- 
drial activity and oxidative capacity. J Biol Chem. 2010;285(29):22619-22629.

20. Wang YX, et al. Regulation of muscle fiber type and running endurance by PPARdelta. PLOS Biol. 2004;2(10):e294.

21. Yamamoto H, et al. NCoR1 is a conserved physiological modulator of muscle mass and oxidative function. Cell. 2011;147(4):827-839.

22. Calo E, Wysocka J. Modification of enhancer chromatin: what, how, and why? Mol Cell. 2013;49(5):825-837.

23. Heinz S, Romanoski CE, Benner C, Glass $\mathrm{CK}$. The selection and function of cell type-specific enhancers. Nat Rev Mol Cell Biol. 2015;16(3):144-154.

24. Cao K, et al. An Mll4/COMPASS-Lsd1 epigenetic axis governs enhancer function and pluripotency transition in embryonic stem cells. Sci Adv. 2018;4(1):eaap8747.

25. Hu D, Gao X, Morgan MA, Herz HM, Smith ER, Shilatifard A. The MLL3/MLL4 branches of the COMPASS family function as major histone H3K4 monomethylases at enhancers. Mol Cell Biol. 2013;33(23):4745-4754.

26. Lai B, Lee JE, Jang Y, Wang L, Peng W, Ge K. MLL3/MLL4 are required for CBP/p300 binding on enhancers and super-enhancer formation in brown adipogenesis. Nucleic Acids Res. 2017;45(11):6388-6403.

27. Lee JE, et al. H3K4 mono- and di-methyltransferase MLL4 is required for enhancer activation during cell differentiation. Elife. 2013;2:e1503.

28. Wang C, et al. Enhancer priming by H3K4 methyltransferase MLL4 controls cell fate transition. Proc Natl Acad Sci USA. 2016;113(42):11871-11876.

29. Jang $\mathrm{Y}$, et al. H3.3K4M destabilizes enhancer H3K4 methyltransferases MLL3/MLL4 and impairs adipose tissue development. Nucleic Acids Res. 2019;47(2):607-620.

30. Wang SP, et al. A UTX-MLL4-p300 Transcriptional Regulatory Network coordinately shapes active enhancer landscapes for eliciting transcription. Mol Cell. 2017;67(2):308-321.e6.

31. Ang SY, et al. KMT2D regulates specific programs in heart development via histone H3 lysine 4 di-methylation. Development. 2016;143(5):810-821.

32. Kim DH, et al. Critical roles of the histone methyltransferase MLL4/KMT2D in murine hepatic steatosis directed by ABL1 and PPAR $\gamma 2$. Cell Rep. 2016;17(6):1671-1682.

33. Rao RC, Dou Y. Hijacked in cancer: the KMT2 (MLL) family of methyltransferases. Nat Rev Cancer. 2015;15(6):334-346.

34. $\mathrm{Ng} \mathrm{SB}$, et al. Exome sequencing identifies MLL2 mutations as a cause of Kabuki syndrome. Nat Genet. 2010;42(9):790-793.

35. Zaidi S, et al. De novo mutations in histonemodifying genes in congenital heart disease. Nature. 2013;498(7453):220-223.

36. Brüning JC, et al. A muscle-specific insulin receptor knockout exhibits features of the metabolic syndrome of NIDDM without altering glucose tolerance. Mol Cell. 1998;2(5):559-569.

37. $\mathrm{He} \mathrm{Y}$, et al. Glutamine synthetase in muscle is required for glutamine production during fasting and extrahepatic ammonia detoxification. J Biol Chem. 2010;285(13):9516-9524.

38. Sun Z, et al. Diet-induced lethality due to deletion of the Hdac3 gene in heart and skeletal muscle. J Biol Chem. 2011;286(38):33301-33309.

39. Black BL, Olson EN. Transcriptional control of muscle development by myocyte enhancer factor-2 (MEF2) proteins. Annu Rev Cell Dev Biol. 1998;14:167-196.

40. Sebastian S, et al. Tissue-specific splicing of a ubiquitously expressed transcription factor is essential for muscle differentiation. Genes Dev. 2013;27(11):1247-1259.

41. Liu J, et al. Coupling of mitochondrial function and skeletal muscle fiber type by a miR-499/ Fnip1/AMPK circuit. EMBO Mol Med. 2016;8(10):1212-1228.

42. van Rooij E, et al. A family of microRNAs encoded by myosin genes governs myosin expression and muscle performance. Dev Cell. 2009;17(5):662-673.

43. Teran-Garcia M, Rankinen T, Koza RA, Rao DC, Bouchard C. Endurance training-induced changes in insulin sensitivity and gene expression. Am J Physiol Endocrinol Metab. 2005;288(6):E1168-E1178.

44. Cheng JC, et al. Adolescent idiopathic scoliosis. Nat Rev Dis Primers. 2015;1:15030.

45. Martínez-Llorens J, et al. Muscle dysfunction and exercise limitation in adolescent idiopathic scoliosis. Eur Respir J. 2010;36(2):393-400.

46. Luciano Rde P, et al. Adolescent idiopathic scolio- sis without limb weakness: a differential diagnosis of core myopathy? BMC Musculoskelet Disord. 2015;16:179.

47. Mannion AF, Meier M, Grob D, Müntener M. Paraspinal muscle fibre type alterations associated with scoliosis: an old problem revisited with new evidence. Eur Spine J. 1998;7(4):289-293.

48. Stetkarova I, Zamecnik J, Bocek V, Vasko P, Brabec K, Krbec M. Electrophysiological and histological changes of paraspinal muscles in adolescent idiopathic scoliosis. Eur Spine J. 2016;25(10):3146-3153.

49. Lee KY, et al. Tbx15 controls skeletal muscle fibre-type determination and muscle metabolism. Nat Commun. 2015;6:8054.

50. Quiat D, et al. Concerted regulation of myofiberspecific gene expression and muscle performance by the transcriptional repressor Sox6.Proc Natl Acad Sci USA. 2011;108(25):10196-10201.

51. Rampalli S, et al. p38 MAPK signaling regulates recruitment of Ash2L-containing methyltransferase complexes to specific genes during differentiation. Nat Struct Mol Biol. 2007;14(12):1150-1156.

52. Zhang M, Zhu B, Davie J. Alternative splicing of MEF2C pre-mRNA controls its activity in normal myogenesis and promotes tumorigenicity in rhabdomyosarcoma cells. J Biol Chem. 2015;290(1):310-324.

53. Adam MP, Hudgins L. Kabuki syndrome: a review. Clin Genet. 2005;67(3):209-219.

54. Fu T, et al. Mitophagy directs muscle-adipose crosstalk to alleviate dietary obesity. Cell Rep. 2018;23(5):1357-1372

55. Liang $X$, et al. Exercise inducible lactate dehydrogenase $\mathrm{B}$ regulates mitochondrial function in skeletal muscle. J Biol Chem. 2016;291(49):25306-25318.

56. Liu L, et al. Coupling of COPII vesicle trafficking to nutrient availability by the IRE1 $\alpha$-XBP1s axis Proc Natl Acad Sci USA. 2019;116(24):11776-11785.

57. Jang Y, Wang C, Zhuang L, Liu C, Ge K. H3K4 Methyltransferase activity is required for MLL4 protein stability. J Mol Biol. 2017;429(13):2046-2054.

58. Wright CE, Haddad F, Qin AX, Bodell PW, Baldwin KM. In vivo regulation of beta-MHC gene in rodent heart: role of $\mathrm{T} 3$ and evidence for an upstream enhancer. Am J Physiol. 1999;276(4):C883-C891. 